\title{
Gemini NIFS survey of feeding and feedback in nearby active galaxies - V. Molecular and ionized gas kinematics
}

\author{
M. Bianchin, ${ }^{1 \star}$ R. A. Riffel ${ }^{\oplus},{ }^{1 \star}$ T. Storchi-Bergmann, ${ }^{2}$ R. Riffel ${ }^{\oplus},{ }^{2}$ D. Ruschel-Dutra ${ }^{(},{ }^{3}$ \\ C. M. Harrison ${ }^{\oplus},{ }^{4}$ L. G. Dahmer-Hahn, ${ }^{5,6}$ V. Mainieri, ${ }^{7}$ A. J. Schönell ${ }^{8}$ and N. Z. Dametto ${ }^{9}$ \\ ${ }^{1}$ Centro de Ciências Naturais e Exatas, Departamento de Física, Universidade Federal de Santa Maria, 97105-900 Santa Maria, RS, Brazil \\ ${ }^{2}$ Instituto de Física, Universidade Federal do Rio Grande do Sul, Av. Bento Gonçalves 9500, 91501-970, Porto Alegre, RS, Brazil \\ ${ }^{3}$ Departamento de Física, Universidade Federal de Santa Catarina, PO Box 476, 88040-900 Florianópolis, SC, Brazil \\ ${ }^{4}$ School of Mathematics, Statistics and Physics, Newcastle University, Newcastle upon Tyne NE1 7RU, UK \\ ${ }^{5}$ Shanghai Astronomical Observatory, Chinese Academy of Sciences, 80 Nandan Road, Shanghai 200030, China \\ ${ }^{6}$ Laboratório Nacional de Astrofísica, Rua dos Estados Unidos 154, CEP 37504-364 Itajubá, MG, Brazil \\ ${ }^{7}$ European Southern Observatory, Karl-Schwarzschild-Strasse 2, D-85748 Garching bei München, Germany \\ ${ }^{8}$ Instituto Federal de Educação, Ciência e Tecnologia Farroupilha, BR 287, Km 360, Estrada do Chapadão, 97760-000 Jaguari, RS, Brazil \\ ${ }^{9}$ Centro de Astronomía (CITEVA), Universidad de Antofagasta, Avenida Angamos 601, Antofagasta, Chile
}

Accepted 2021 November 26. Received 2021 November 25; in original form 2021 August 21

\begin{abstract}
We study the gas distribution and kinematics of the inner kpc of six moderately luminous $\left(43.43 \leq \log L_{\text {bol }} \leq 44.83\right)$ nearby $(0.004 \leq z \leq 0.014)$ Seyfert galaxies observed with the Near-infrared Integral Field Spectrograph (NIFS) in the $J(1.25 \mu \mathrm{m})$ and $K(2.2 \mu \mathrm{m})$ bands. We analyse the most intense emission lines detected on these spectral wavebands: [Fe II] $1.2570 \mu \mathrm{m}$ and $\mathrm{Pa} \beta$, which trace the ionized gas in the partially and fully ionized regions, and $\mathrm{H}_{2} 2.1218 \mu \mathrm{m}$, which traces the hot $(\sim 2000 \mathrm{~K})$ molecular gas. The dominant kinematic component is rotation in the disc of the galaxies, except for the ionized gas in NGC 5899 that shows only weak signatures of a disc component. We find ionized gas outflow in four galaxies, while signatures of $\mathrm{H}_{2}$ outflows are seen in three galaxies. The ionized gas outflows display velocities of a few hundred $\mathrm{km} \mathrm{s}^{-1}$, and their mass outflow rates are in the range $0.005-12.49 \mathrm{M}_{\odot} \mathrm{yr}^{-1}$. Their kinetic powers correspond to $0.005-0.7$ per cent of the active galactic nuclei (AGN) bolometric luminosities. Besides rotation and outflows signatures in some cases, the $\mathrm{H}_{2}$ kinematics also reveals inflows in three galaxies. The inflow velocities are 50-80 km s${ }^{-1}$ and the mass inflow rates are in the range $1-9 \times 10^{-4} \mathrm{M}_{\odot} \mathrm{yr}^{-1}$ for hot molecular gas. These inflows might be only the hot skin of the total inflowing gas, which is expected to be dominated by colder gas. The mass inflow rates are lower than the current accretion rates to the AGN, and the ionized outflows are apparently disturbing the gas in the inner kpc.
\end{abstract}

Key words: galaxies: active-galaxies: kinematics and dynamics - galaxies: nuclei-galaxies: Seyfert.

\section{INTRODUCTION}

The presence of a supermassive black hole (SMBH) at the centre of every galaxy with a spheroidal component is a well-accepted paradigm of extragalactic astronomy (Kormendy \& Ho 2013; van den Bosch 2016), although the absence of a bulge does not imply the absence of a SMBH (Kormendy, Bender \& Cornell 2011). Correlations between the SMBH and host galaxy properties, e.g. the $M-\sigma_{\star}$ relation (Magorrian et al. 1998; Ferrarese \& Merritt 2000; Gebhardt et al. 2000; Caglar et al. 2020), have been studied over more than two decades and such correlations indicate that the growth of SMBHs and their host galaxies are coupled. The galaxy, or interactions with neighbouring galaxies, provides the fuel necessary to feed the SMBH (Storchi-Bergmann \& Schnorr-Müller 2019) giving origin to active galactic nuclei (AGN). Once triggered, the AGN then release energy in the form of radiation, gas outflows,

^E-mail: marina.bianchin@acad.ufsm.br (MB); rogemar.ufsm.br (RAF) or jets of particles that can affect the evolution of the host galaxy creating the so-called AGN feedback (e.g. Fabian 2012).

Galactic scale feedback from AGN is now understood as one of the main mechanisms acting on star formation suppression of a galaxy (Harrison 2017), transforming it from star forming to quiescent. Also, the addition of AGN feedback into cosmological models explains the observed luminosity function of galaxies (Benson et al. 2003) and reproduces a realistic galaxy population (Bower et al. 2006; Nelson et al. 2019; Pillepich et al. 2019).

Theoretical models do predict that AGN-driven outflows can suppress the star formation in the host galaxy (Granato et al. 2004; Zubovas \& Bourne 2017; Costa, Pakmor \& Springel 2020) and the relativistic jets can disturb the gas in the interstellar medium (ISM) preventing star formation (Mukherjee et al. 2018). On the other hand, depending on the power of the AGN feedback, some simulations also predict an enhancement of the star formation (Hopkins 2012; Nayakshin \& Zubovas 2012; Zubovas et al. 2013; Bieri et al. 2016; Zubovas \& Bourne 2017) or even new stars being formed inside the outflow (Ishibashi \& Fabian 2012; Zubovas et al. 2013; El-Badry 
et al. 2016). Because of the lack of strong observational constraints, the AGN feedback is usually included in the simulations in an ad hoc way (Nelson et al. 2019).

The recent review by Veilleux et al. (2020) states that molecular outflows $\left(T<10^{4} \mathrm{~K}\right)$ are the main drivers of negative feedback, i.e. quenching of star formation in a galaxy. A way to search for molecular gas outflows is by observing the $\mathrm{H}_{2}$ rotationalvibrational transitions in the near-infrared (near-IR) using integral field spectroscopy (IFS; Emonts et al. 2017; Riffel et al. 2020; Riffel 2021), where the hot $(2000 \mathrm{~K})$ molecular phase of the gas can be mapped even though it represents just the 'tip of the iceberg' (only the heated part) of a much larger cold gas reservoir, which is usually mapped and studied through CO emission lines detected at millimetric wavelengths. Although evidence has been found of cold molecular gas outflows that could, in principle, suppress star formation (Lutz et al. 2020), the estimate of the mass outflow rate relies on the conversion factor from $\mathrm{CO}$ to $\mathrm{H}_{2}$ that is highly uncertain (Veilleux et al. 2020). Thus, it is important to directly map the $\mathrm{H}_{2}$ emission, even if this is only possible in its hottest phase, probed by the near-IR emission lines.

Another key aspect of AGN is how the SMBH becomes active and remains at this phase through time. Storchi-Bergmann \& SchnorrMüller (2019) revised the main processes involved in the SMBH feeding. At the largest scales, galaxy mergers (e.g. Hopkins, Kocevski \& Bundy 2014; Storchi-Bergmann et al. 2018) and chaotic cold accretion (Gaspari, Ruszkowski \& Oh 2013; Diniz et al. 2017), also called cooling flows, are important phenomena. In the case of galactic scales, disc instabilities cause the gas to lose angular momentum and move towards the centre. The bars can also trap the gas in rings surrounding the galaxy centre, but they do not seem to feed the AGN directly, as no difference between the accretion rates of barred and non-barred galaxies is observed (Galloway et al. 2015). Using Hubble Space Telescope (HST) imaging, Martini et al. (2003b) showed that the presence of dust is common among AGN hosts (Martini et al. 2003b) and it can be related to the feeding of the $\mathrm{SMBH}$, but no difference is found comparing active and non-active samples. The time-scale for the flow of gas through the dust lanes to centre may be larger than the AGN activity cycle, explaining the lack of significant difference of dust distribution between active and nonactive galaxies. In a subsequent work, Simões Lopes et al. (2007) have shown that although there is indeed no difference between AGN and non-AGN for late-type galaxies, there is a marked difference in early-type galaxies: most early-type AGN show dust, compared to only 25 per cent of the control galaxies. This suggests an external origin for the gas in early-type AGN.

More direct observations of the AGN fuelling are based on studies of the gas dynamics in the inner kiloparsec of AGN hosts, using IFS that has been successful in mapping gas streaming towards the centre of galaxies (e.g. Fathi et al. 2006; Storchi-Bergmann et al. 2007; Riffel et al. 2008; Müller Sánchez et al. 2009; Riffel, Storchi-Bergmann \& Winge 2013b; Diniz et al. 2015; SchnorrMüller et al. 2017b). Signatures of gas inflows are observed in multigas phases: (i) the cold molecular gas $(\sim 100 \mathrm{~K})$ can be traced at millimetric wavelengths with facilities like the Atacama Large Millimeter/submillimeter Array (ALMA) using spatially resolved observations of the CO transitions (e.g. Combes et al. 2013); (ii) the hot molecular gas $(\sim 2000 \mathrm{~K})$ that is observed in the near-IR and can be mapped through the $\mathrm{H}_{2}$ emission lines in the $K$ band (Riffel et al. 2013b; Diniz et al. 2015), where the strongest is the $\mathrm{H}_{2}(1-0)$ $\mathrm{S}(1) 2.1218 \mu \mathrm{m}$ emission line; (iii) the hot ionized gas $(\sim 10000 \mathrm{~K})$ using optical emission lines like the [N II] (e.g. Schnorr-Müller et al. 2017a). The inflow rates are typically $\sim 1 \mathrm{M}_{\odot} \mathrm{yr}^{-1}$, which are three orders of magnitudes higher than the SMBH accretion rates, meaning the gas can feed the AGN and accumulate at the centre and form stars over an AGN duty-cycle (Storchi-Bergmann \& Schnorr-Müller 2019). Other studies of the inner region of active galaxies found that the molecular hydrogen emission usually originates from the gas in the disc (Barbosa et al. 2014; Riffel, Storchi-Bergmann \& Riffel 2015), meaning its kinematics tend to be similar to the stellar one, but in some cases streaming motions towards the centre may also be observed (Riffel et al. 2008, 2013b; Diniz et al. 2015). Hot $\mathrm{H}_{2}$ outflows are also observed in some nearby AGN hosts at scales of a few hundred parsecs (e.g. Davies et al. 2014; Ramos Almeida et al. 2017; Riffel et al. 2020; Riffel 2021).

Our group [the AGN Integral Field Spectroscopy (AGNIFS) group] has been using observations with the Gemini Near-infrared Integral Field Spectrograph (NIFS) to map the gas and stellar properties in the inner kiloparsec of nearby active galaxies. The main sample is composed of 20 objects and is described in Riffel et al. (2018). So far, 16 objects have been observed with NIFS or have Very Large Telescope (VLT)-Spectrograph for INtegral Field Observations in the Near Infrared (SINFONI) archival data. The observation of one galaxy is on queue for this semester and we expect to conclude the observations of the remaining three objects in 2022, before NIFS is decommissioned from the observatory. The gas kinematics of both ionized and molecular gas usually presents a rotating disc component. Outflows are commonly observed in ionized gas and scarce in molecular gas that, in some cases, besides the rotation, presents signatures of inflows (Storchi-Bergmann et al. 2010; Riffel \& Storchi-Bergmann 2011a,b; Riffel et al. 2013b, 2020; Riffel, Storchi-Bergmann \& Riffel 2014; Schönell et al. 2014, 2017, 2019; Diniz et al. 2015, 2019; Gnilka et al. 2020). Our previous work (Schönell et al. 2019) shows flux distribution and kinematic maps of the same galaxies we are studying here. The biggest difference between this paper and the previous is that now we disentangle the different emission components (see Section 3.1) and quantify the non-rotational motions, like outflows and inflows. Such features were just suggested, and not investigated in deeper detail, in Schönell et al. (2019).

In this paper, we continue our series of works aimed at investigating the AGN feeding and feedback processes. We analyse the ionized and molecular gas kinematics of six active galaxies observed with Gemini NIFS in the $J$ and $K$ bands. In Section 2, we present our sample and a summary of the observations and the data reduction procedure. Section 3.1 presents the method used to fit the emission line profiles, while in Section 3.2, we present the resulting flux, velocity, and velocity dispersion maps. We compare our results with those from the literature and further analyse our results in Section 4, summarizing the impact of the gas inflows and outflows on the galaxies in Section 5. Our conclusions are presented in Section 6. Throughout this paper we adopt $h=0.7, \Omega_{\mathrm{m}}=0.3$, and $\Omega_{\lambda}=0.7$ cosmology.

\section{THE SAMPLE AND DATA}

\subsection{The sample of active galaxies}

The galaxies in this paper are part of the AGNIFS sample (Riffel et al. 2018), composed of Seyfert galaxies observed with the NIFS on the Gemini North Telescope. This sample was selected by adopting the following criteria: (i) X-ray luminosity $L_{\mathrm{X}}>10^{41.5} \mathrm{erg} \mathrm{s}^{-1}$ in the 14$195 \mathrm{keV}$ band in the 105-month Swift-Burst Alert Telescope (BAT) catalogue (Oh et al. 2018); (ii) $z \leq 0.015$; and (iii) $-30^{\circ}<\delta<$ $73^{\circ}$, making them accessible to NIFS. An additional criterion - the 
presence of extended [O III] optical emission previously detected in the galaxies (see Riffel et al. 2018 and references therein) - was added to guarantee these sources present extended emission from the ionized gas in the near-IR. The hard X-ray luminosities are particularly suited to the selection of AGN because, in principle, this radiation is not obscured in the line of sight as would happen in optical wavebands up to Compton thick densities (Ricci et al. 2015). The redshift criterion guarantees we reach tens of parsec in spatial resolution, necessary for resolving inflows and compact outflows.

In this work, we study six galaxies selected from the AGNIFS sample: NGC 788, NGC 3516, NGC 5506, NGC 3227, NGC 5899, and Mrk 607. These galaxies are among the 50 per cent most luminous ones without previous studies of their kinematics using NIFS. The other 10 galaxies have already been studied by our group or are currently being analysed (Riffel et al., in preparation). They cover a luminosity range of $43.4<\log L_{\text {bol }}\left(\mathrm{erg} \mathrm{s}^{-1}\right)<44.8$ and a redshift range of $0.004<z<0.014$. We obtained $J(1.25 \mu \mathrm{m})$ band data cubes for all galaxies, $K(2.20 \mu \mathrm{m})$ for NGC 788, NGC 3516 , and NGC 5506, and $K_{\text {long }}(2.30 \mu \mathrm{m})$ for NGC 3227, NGC 5899, and Mrk 607. In the $K_{\text {long }}$ grating, some molecular hydrogen transitions are not available, but this does not affect the analysis we perform here, which is focused on the analysis of the strongest emission lines from the molecular and ionized gas.

The NIFS data of these galaxies have been previously used in two studies. In Schönell et al. (2019), we presented the data and gas distribution and kinematics based on emission line fitting with Gauss-Hermite functions (for details on the differences between the present and previous fitting methods see Section 3.1). We also present the spatial distributions of ionized and molecular gas. In Riffel et al. (2021b), we analyse the gas excitation concluding that thermal processes, such as shocks due to gas outflows, are the main source of excitation to both [Fe II] and $\mathrm{H}_{2}$.

\subsection{Near-IR observations and data}

The NIFS is composed of 29 slitlets with a width of 0.103 arcsec and a height of 3.0 arcsec that together create a field of view (FoV) of $3 \times 3 \operatorname{arcsec}^{2}$. The spatial sampling along each slitlet is 0.042 arcsec. At the redshifts of the galaxies studied here, the NIFS FoV covers less than $1 \mathrm{kpc}$ (see the 1 arcsec scale on the bottom left-hand corner of the continuum maps in Figs 1-6). All observations were carried out between 2012 and 2016 (Observing IDs: GN-2012B-Q-45, GN2013A-Q-48, GN-2015A-Q-3, GN-2015B-Q-29, and GN-2016A-Q6 ) and used the NIFS adaptive optics system ALTtitude conjugate Adaptive optics for the InfraRed (ALTAIR). ALTAIR uses a laser or natural star to guide the observations and correct for the seeing effect. For the $J$ - and $K$-band observations we obtain angular resolutions of 0.13-0.15 and 0.12-0.18 arcsec, respectively, that correspond to a scale of $19-36$ and $22-36 \mathrm{pc}$ in the $J$ and $K$ bands at the distance of the galaxies. The angular resolutions were estimated from the full width at half-maximum (FWHM) of the telluric standard star for the type 2 AGN and from the flux distributions of the broad components of the $\mathrm{Pa} \beta$ and $\mathrm{Br} \gamma$ emission lines for the type 1 objects. The spectral resolutions of $\sim 1.8 \AA$, in the $J$ band, and $\sim 3.2 \AA$, in the $K$ band, corresponding to $\approx 45 \mathrm{~km} \mathrm{~s}^{-1}$, make the NIFS data appropriate to characterize the gas kinematics.

The data reduction procedure, explained in detail in Schönell et al. (2019), uses the Gemini NIFS IRAF packages. Several exposures of each galaxy are performed (see table 1 in Schönell et al. 2019) and, in each of them, the main reduction steps are the flat-fielding, sky subtraction, s-distortion correction, and wavelength and flux calibrations. The data cubes for individual exposures were mean combined using the peak of the continuum emission as the reference for astrometry creating a final data cube with a pixel size of 0.05 arcsec for each band for all galaxies.

\section{MEASUREMENTS AND TWO-DIMENSIONAL MAPS}

\subsection{Measurements}

The $J$ and $K$-bands spectra of nearby active galaxies are rich in emission lines from ionized species and molecular hydrogen (Riffel, Rodríguez-Ardila \& Pastoriza 2006). Here we focus our analysis on the strongest emission lines in active galaxies - [Fe II $] 1.2570 \mu \mathrm{m}$, $\mathrm{Pa} \beta \lambda 1.282 \mu \mathrm{m}$, and $\mathrm{H}_{2} 2.1218 \mu \mathrm{m}$, which represent distinct gas phases (Rodríguez-Ardila, Riffel \& Pastoriza 2005; Riffel et al. 2006, 2013a, 2019). In the specific case of the $\mathrm{H}_{2} 2.1218 \mu \mathrm{m}$, this line is also selected because it is less affected by telluric absorptions and is within the observed spectral $K$-band range in the six galaxies.

These emission lines have distinct physical origins: the [Fe II] $1.2570 \mu \mathrm{m}$ emission originates in the partially ionized region, where the flux of ionizing radiation is not sufficient to fully ionize the gas, has an ionization potential of $7.9 \mathrm{eV}$, and is a good tracer of AGN outflows (Storchi-Bergmann et al. 2010; Barbosa et al. 2014); the $\mathrm{Pa} \beta$ is a recombination line that traces the ionized gas emission; and the $\mathrm{H}_{2}$ emission lines map the hot skin of the densest regions associated with the cold molecular gas. Because of the common origin, $\mathrm{Pa} \beta$ and $\mathrm{Br} \gamma$ emission lines have the same spatial distribution, i.e. trace the same gas. We choose the former because it is more intense than the latter.

We fitted the emission line profiles in each spaxel using the PYTHON-based package IFSCUBE that is designed to handle data cubes from IFS data or single spectrum observations (Ruschel-Dutra $\&$ de Oliveira 2020). To fit the emission lines we used the CUBEFIT module that allows the fit of one or more Gaussian or Gauss-Hermite functions to the observed emission line profiles. Initial guesses and/or bounds for each of the fitting parameters can be provided by the user: the amplitude $(A)$, centroid velocity $(V)$, velocity dispersion $(\sigma)$, and the $h 3$ and $h 4$ moments specifically for the Gauss-Hermite fit.

We also removed the continuum contribution with a low-order polynomial. As we selected only the region close to the line to be fitted, in almost all of our fits we used a first-degree polynomial to represent the continuum. The exceptions are the third-order polynomial in both the $J$ - and $K$-band fits of Mrk 607 and in the $K$-band fit of NGC 5899, and the fourth-order polynomial for the $J$ band in NGC 3516.

In our sample, three galaxies are classified as type 1 Seyfert (Sy1; Oh et al. 2018): NGC 3227, NGC 3516, and NGC 5506, meaning the broad line region (BLR) of the AGN is detected. The BLR is identified as a broad component $\left(\sim 1000 \mathrm{~km} \mathrm{~s}^{-1}\right)$ superimposed to the other components of the $\operatorname{Pa} \beta$ and $\operatorname{Br} \gamma$, in the $J$ and $K$-bands spectral range. Since the BLR is not spatially resolved, their line profiles were fitted by a Gaussian function with fixed velocity and velocity dispersion in all spaxels they appeared, as measured from the nuclear spectrum of each galaxy.

In previous works with these galaxies different strategies to fit the emission lines have been adopted. In Schönell et al. (2019), Gauss-Hermite functions were chosen because the emission line profiles deviated from a Gaussian function. A different approach was adopted in Riffel et al. (2021b) where up to three Gaussian functions were fitted to each emission line. Both approaches aimed 

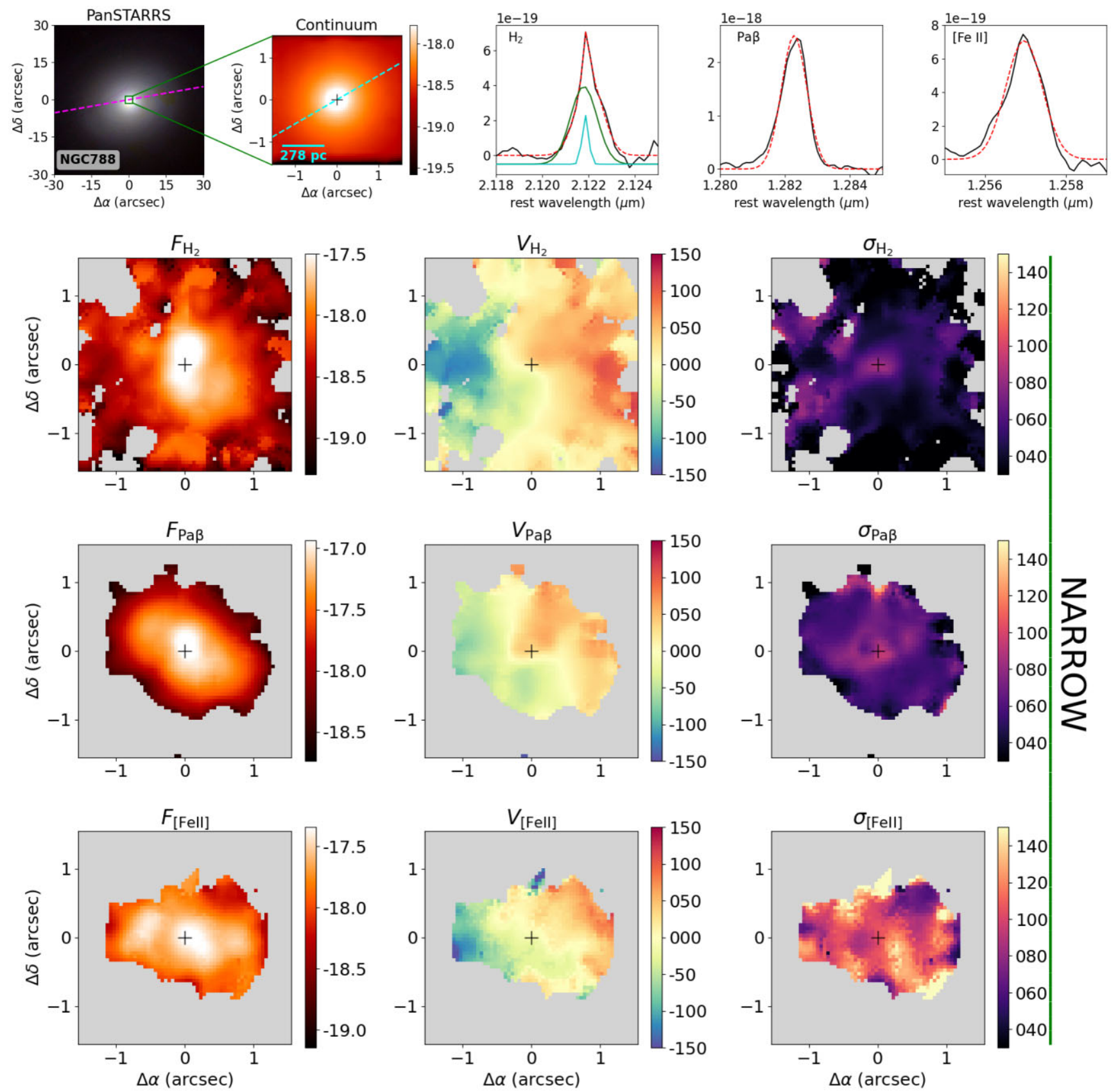

Figure 1. NGC 788. First row shows, in the left-hand panel, a Panoramic Survey Telescope and Rapid Response System (Pan-STARRS) large-scale $\left(1 \times 1 \operatorname{arcmin}^{2}\right)$ colour image in the $y, i$, and $g$ bands. The green square indicates the NIFS FoV $\left(3 \times 3 \operatorname{arcsec}^{2}\right)$ that is zoomed-in in the continuum image from a $100 \AA$ window centred at $2.14 \mu \mathrm{m}$ in the $K$ band shown in the adjacent panel. The magenta and cyan dashed lines indicate the orientation of the galaxy major axis and stellar kinematics position angle (Riffel et al. 2017), respectively. Examples of the $\mathrm{H}_{2}, \mathrm{~Pa} \beta$, and [Fe II] profile fits are shown in the three panels to the right. The continuum contribution is subtracted from the observed (black continuous line) and modelled (red dashed line) spectra. In the $\mathrm{H}_{2}$ panel, the narrow component is represented in green and the spurious sky component in blue. Bottom three rows: 2D maps obtained from the Gaussian fit to the emission lines. The first column shows the flux distribution in units of $\mathrm{erg} \mathrm{s}^{-1} \mathrm{~cm}^{-2} \AA^{-1}$ for the $\mathrm{H}_{2} 2.1218 \mu \mathrm{m}, \mathrm{Pa} \beta$, and [Fe II] $1.2570 \mu \mathrm{m}$. The second and third columns show the velocity and velocity dispersion maps in $\mathrm{km} \mathrm{s}^{-1}$. The black cross indicates the position of the nucleus (peak of the continuum flux as in Schönell et al. 2019). The grey regions correspond to positions with line amplitudes smaller than $3 \sigma$ of the continuum flux adjacent to the line or with spurious measurements. In all maps the north is up and east to the left.

at the best representation of the observed line profiles and no physical meaning to the components was attributed. In this paper, we fit up to three Gaussian functions to the profile of each emission line. The criterion to decide which model provides the best representation of the observed spectral features is based on the following procedure. We calculate the mean of the residuals, observed spectra - model divided by the standard deviation for each emission line within a window of $1000 \mathrm{~km} \mathrm{~s}^{-1}$. We then select as the best model the one that gives the lowest value for this residual. An special case is Mrk 607, where the emission line profiles have a clear double-peak structure and it has a well-known equatorial outflow (Freitas et al. 2018), so our procedure tried to address this. Using this strategy and previous 

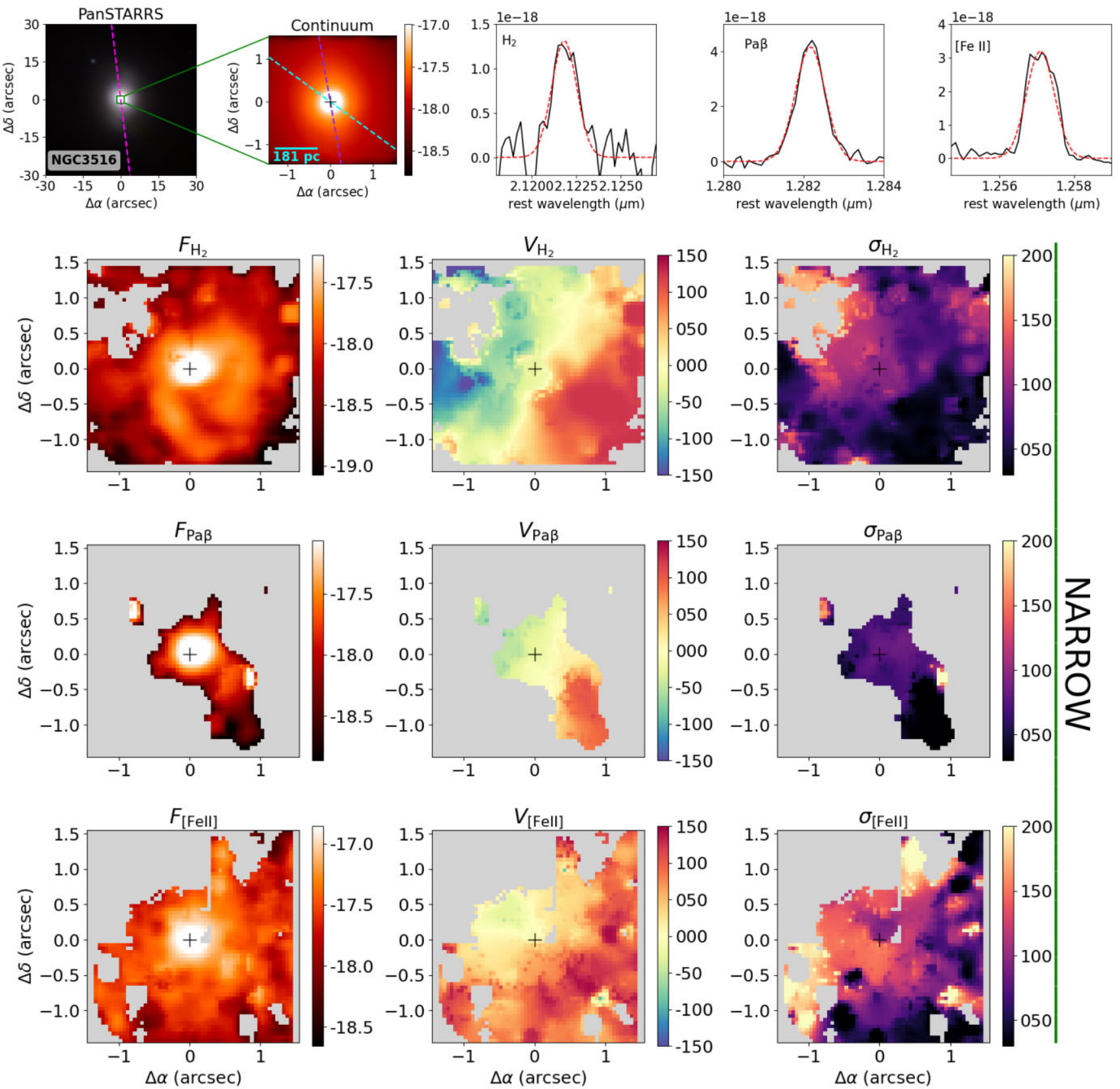

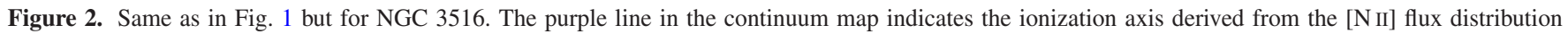
(Ruschel-Dutra et al. 2021). The broad line region (BLR) component to the $\mathrm{Pa} \beta$ has been subtracted from the observed and modelled spectra.

results from the literature on these galaxies, we fitted the following number of Gaussian functions to the [Fe II], $\mathrm{Pa} \beta$, and $\mathrm{H}_{2} 2.1218 \mu \mathrm{m}$ emission lines.

(i) For NGC 788 and NGC 3516, a single Gaussian component can reproduce the observed profiles at all locations. For NGC 788 a sky feature close to the $\mathrm{H}_{2} 2.1218 \mu \mathrm{m}$ significantly affected the maps presented in Schönell et al. (2019). We have now taken care of this feature as follows. The line profile was fitted by two components: one representing the $\mathrm{H}_{2}$ emission and the other, with fixed width $\left(\sigma=20 \mathrm{~km} \mathrm{~s}^{-1}\right)$ and centred at $2.1218 \mu \mathrm{m}$ in all spaxels, for the sky contamination. Since the sky feature is just a contamination, we consider that the $\mathrm{H}_{2}$ is reproduced by a single Gaussian function. (ii) For NGC 5506 and NGC 3227, the [Fe II] $1.2570 \mu \mathrm{m}$ and $\mathrm{Pa} \beta$ line profiles were fitted with two Gaussian curves each, while the $\mathrm{H}_{2} 2.1218 \mu \mathrm{m}$ is well reproduced by a single Gaussian function at all locations.

(iii) Two Gaussian components are needed to reproduce each emission line of NGC 5899.

(iv) For Mrk 607, the emission lines are well reproduced at most locations by a single Gaussian curve tracing the emission of gas in the galaxy disc, except for the inner 0.5 arcsec where three components are present, one due to the disc and two produced by an equatorial outflow previously observed in spectra of this galaxy (Freitas et al. 2018). The same strategy has already been adopted to describe the equatorial outflow in NGC 5929 (Riffel et al. 2015). 

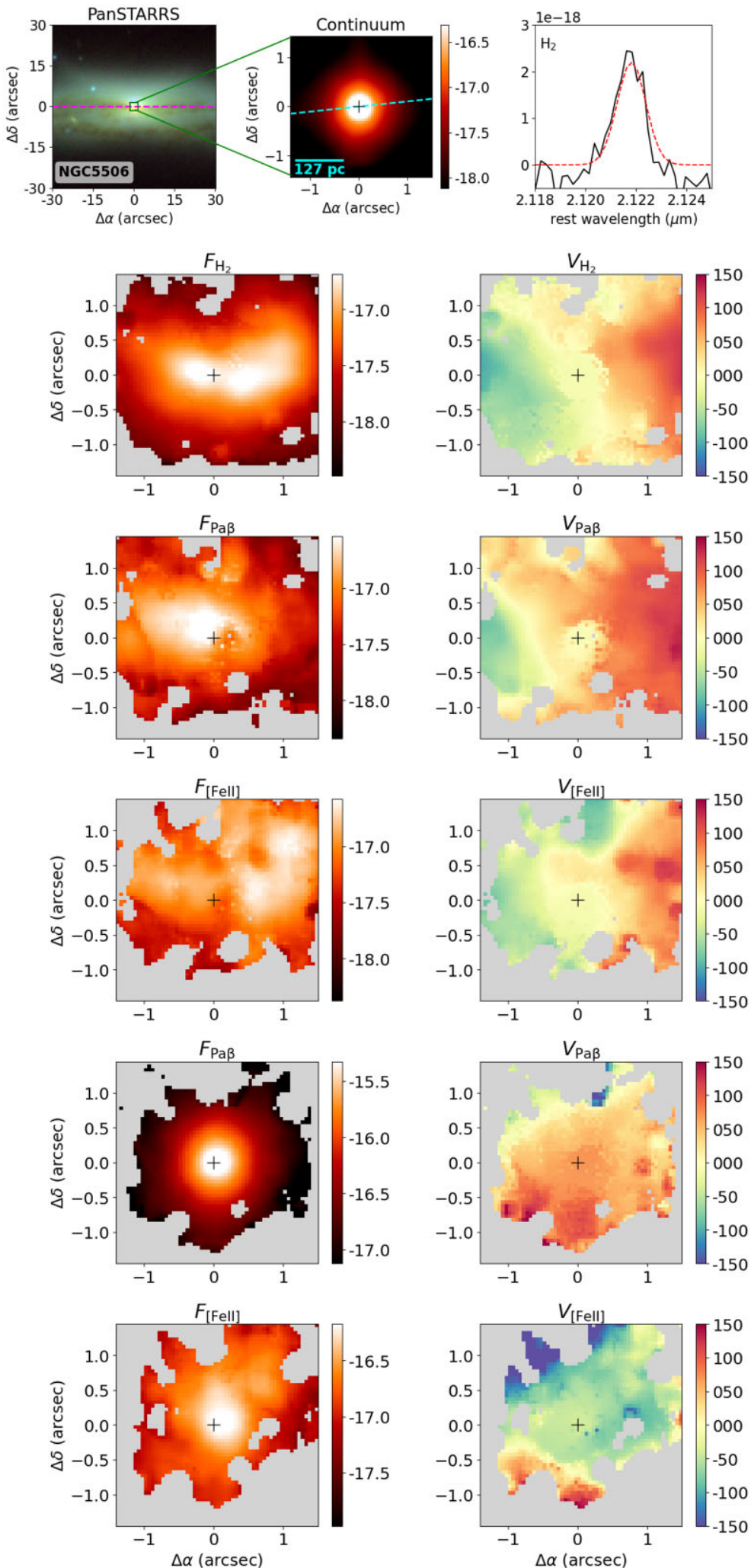
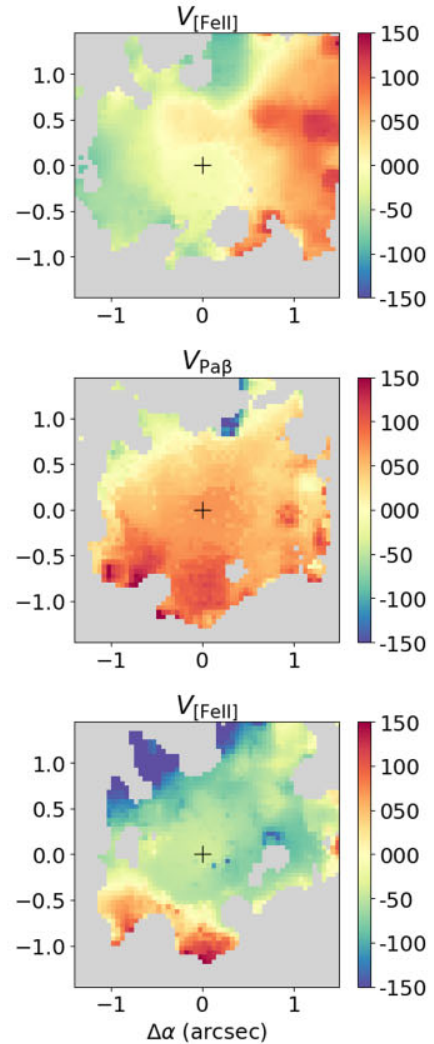
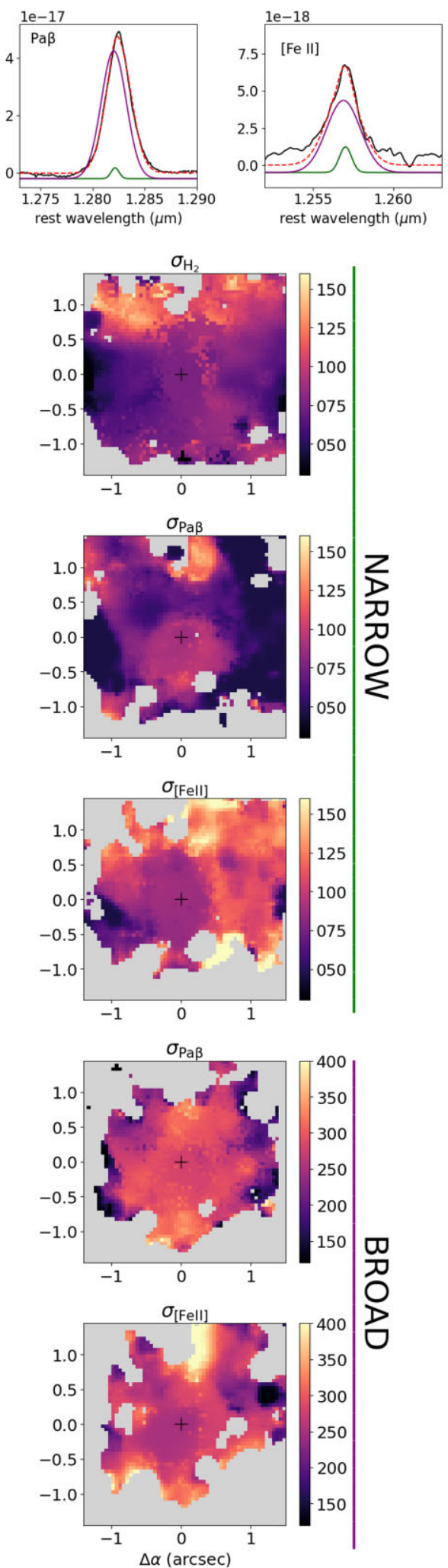

Figure 3. Same as in Fig. 1, but for the narrow and broad components of NGC 5506. In the fits examples the narrow and broad components of Pa $\beta$ and [Fe II] are represented in green and purple, respectively. The broad line region (BLR) component to the $\mathrm{Pa} \beta$ has been subtracted from the observed and modelled spectra.

In the cases where two Gaussians are necessary, one is broad $(\sigma$ $\left.\sim 400 \mathrm{~km} \mathrm{~s}^{-1}\right)$ and one is narrow $\left(\sigma \sim 100 \mathrm{~km} \mathrm{~s}^{-1}\right)$. Examples of the fits, extracted from the nuclear spaxel, are presented in the first line of Figs 1-6.

\subsection{Flux and kinematic maps}

In the top left-hand panel of Figs 1-6, we present the Panoramic Survey Telescope and Rapid Response System (Pan-STARRS) $g$, 

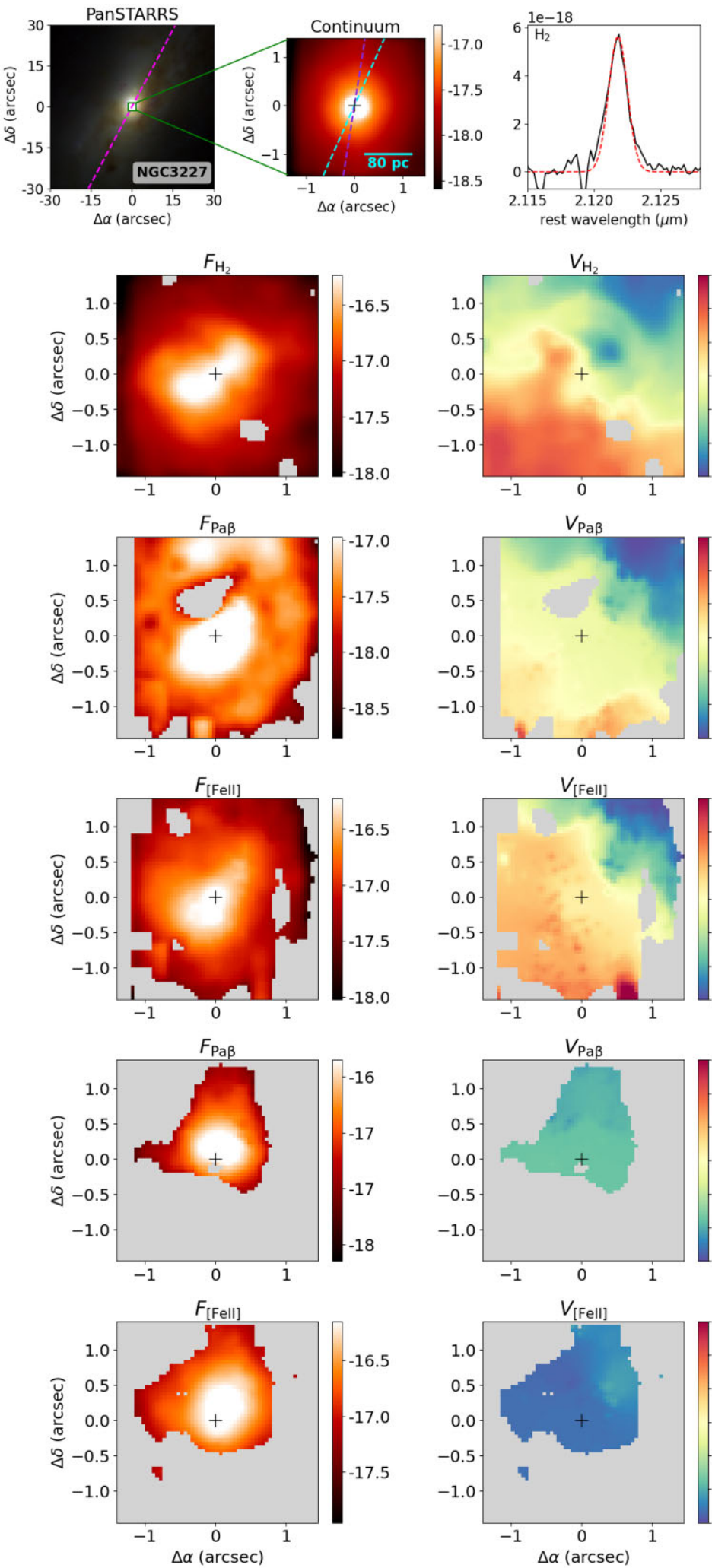
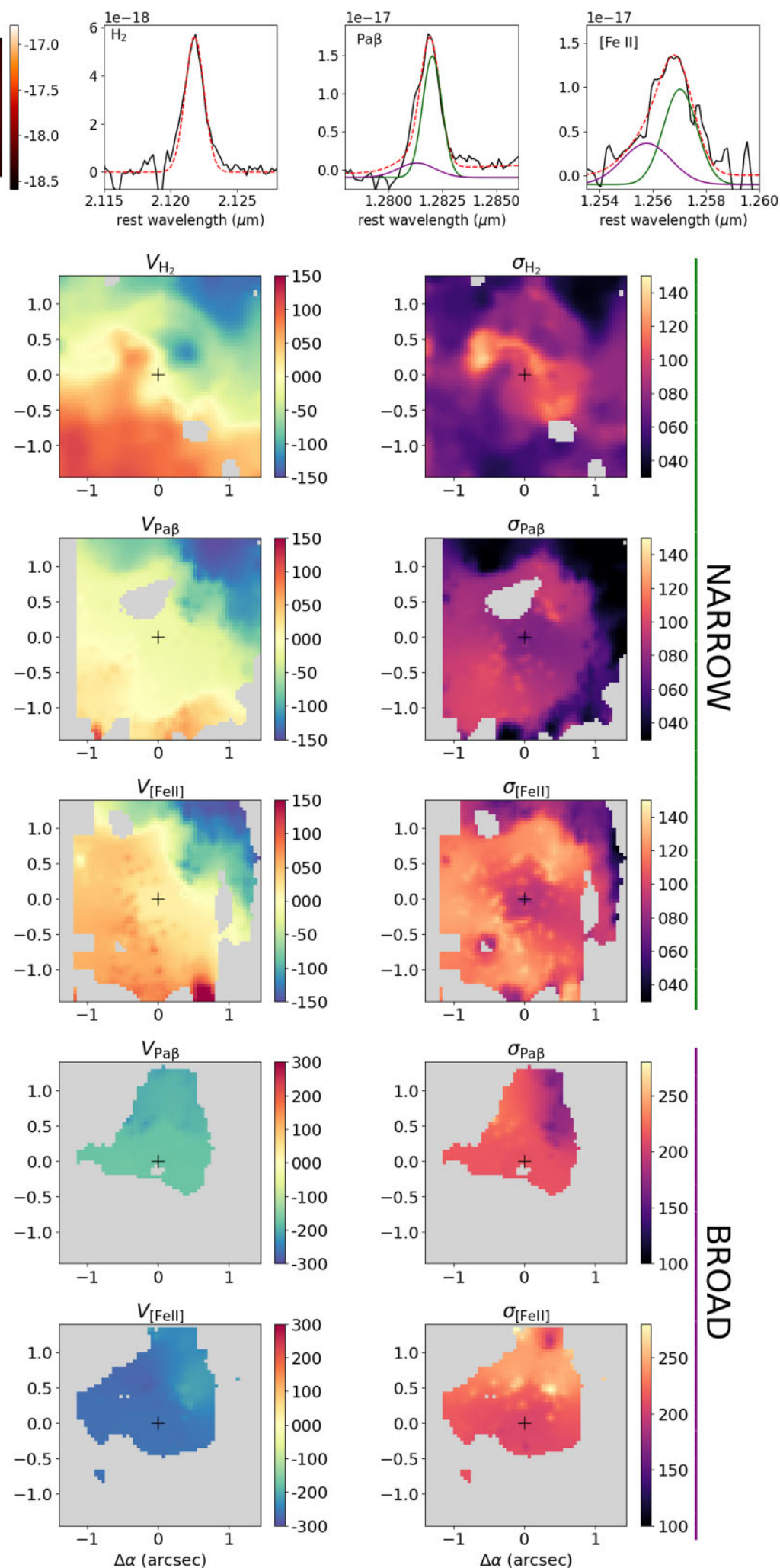

Figure 4. Same as in Fig. 1, but for the narrow and broad components of NGC 3227. In the fits examples the narrow and broad components of Pa $\beta$ and [Fe II] are represented in green and purple, respectively. The broad line region (BLR) component to the $\mathrm{Pa} \beta$ has been subtracted from the observed and modelled spectra.

$i$, and $y$ composite image (Chambers et al. 2019; Flewelling et al. $2020)$ in large scale $\left(1 \times 1 \operatorname{arcmin}^{2}\right)$ for each galaxy in our sample. To the right, we present an image of the continuum, obtained directly from the NIFS data cube in a region free of emission lines, centred at $2.14 \mu \mathrm{m}$, by calculating the mean of the spectra in a window of $100 \AA$. The dashed lines in these two images indicate the orientation of the galaxy major axis and the orientation of the line of the nodes of the stellar velocity field (Riffel et al. 2017). 

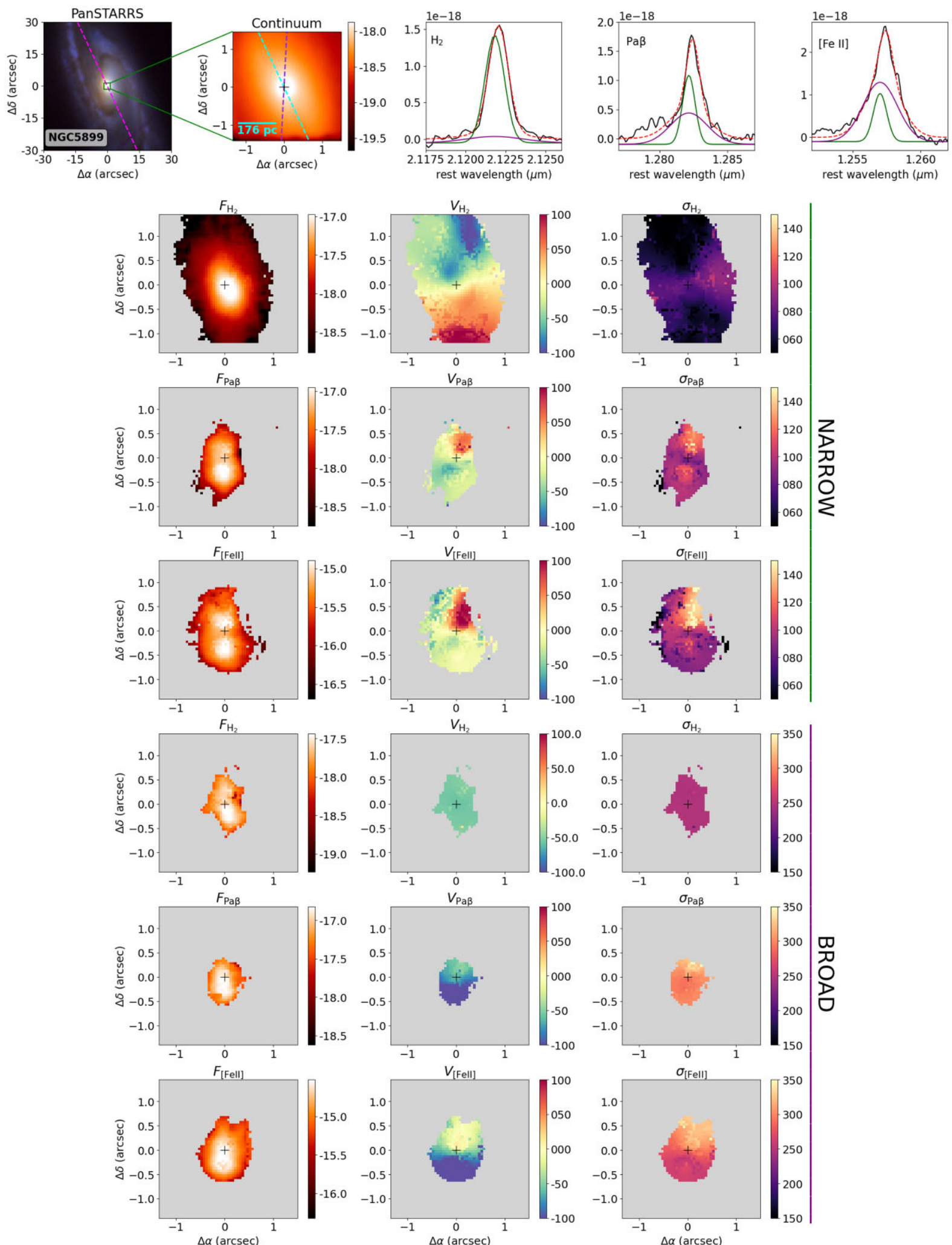

Figure 5. Same as in Fig. 1, but for the narrow and broad components of NGC 5899. In the fits examples the narrow and broad components of $\mathrm{H}_{2}$, $\mathrm{Pa} \beta$, and [Fe II] are represented in green and purple, respectively.

The other three panels show examples of the $\mathrm{H}_{2} 2.1218 \mu \mathrm{m}, \mathrm{Pa} \beta$, and [Fe II] $1.2570 \mu \mathrm{m}$ emission line fits, extracted from the spaxel at the peak of the continuum emission. The black continuous line is the observed and the red dashed line the modelled spectra. For clarity in the plots, the continuum contribution was subtracted from both. When more than one Gaussian function was fitted, we present the individual components as coloured continuous lines: green for narrow, purple for broad, and red and blue for the redshifted and 

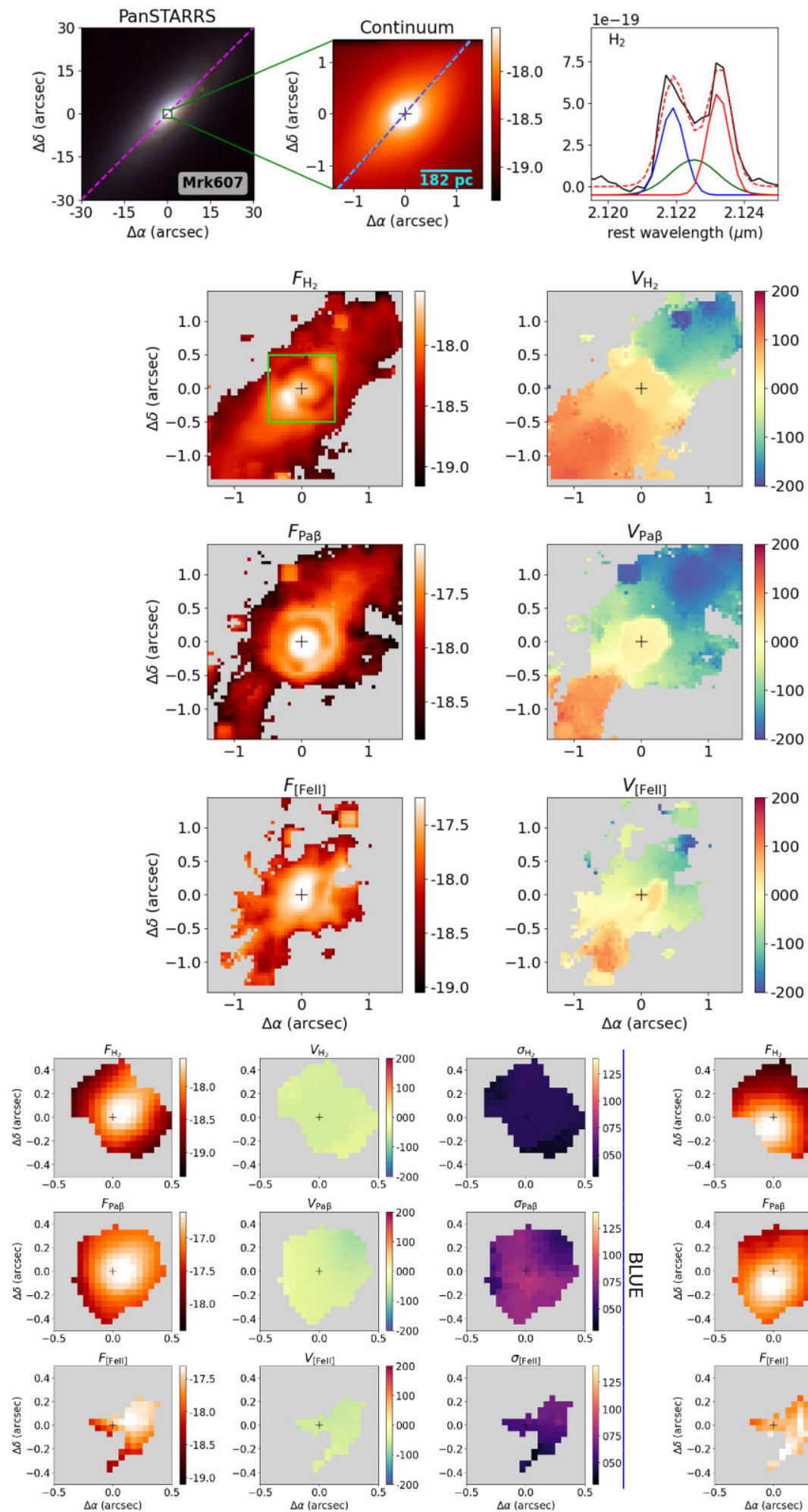
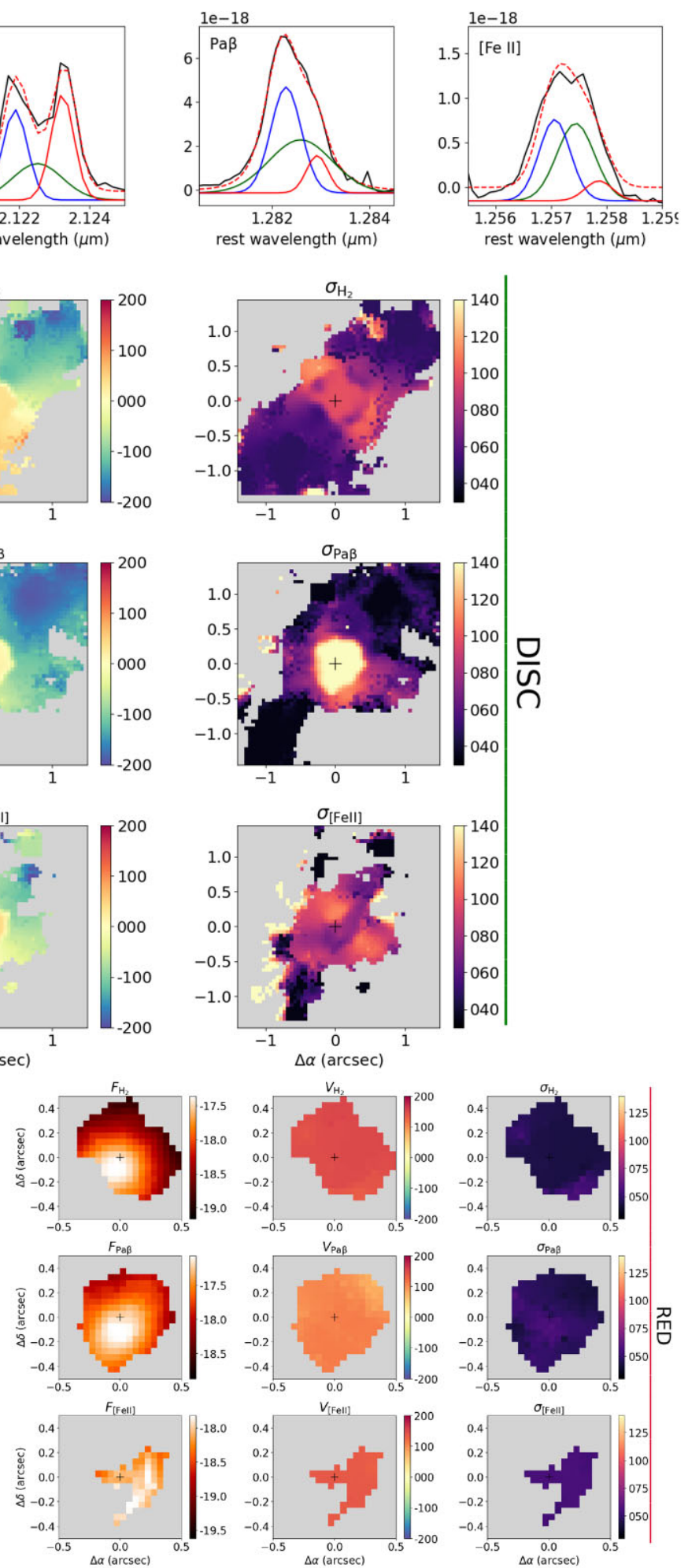

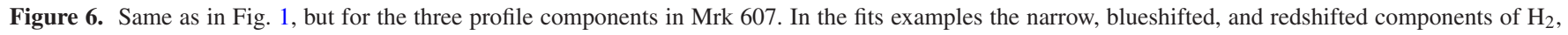

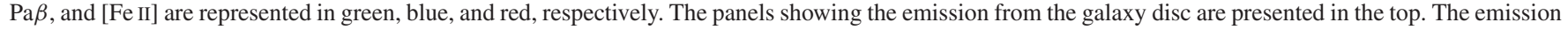

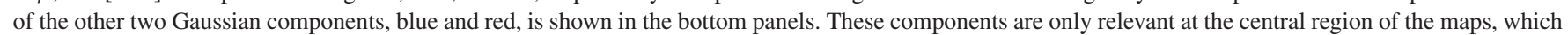
are thus zoomed in to show only the inner $1 \times 1 \mathrm{arcmin}^{2}$, indicated as a square in the top left-hand panel of the disc component.

blueshifted components in Mrk 607. For all galaxies, the nuclear spaxel presents the most complex emission line profiles and, as can be observed from these figures, the adopted models can properly reproduce the observed profiles.
The emission line fitting was performed over the whole NIFS FoV producing two-dimensional (2D) maps for all the fitted parameters. In Figs 1-6, we also present 2D maps for the flux, velocity (after the subtraction of the systemic velocity, defined as the velocity 
obtained by fitting the $\mathrm{H}_{2}$ velocity field by a rotation disc model - see Section 4.1), and velocity dispersion (corrected for the instrumental broadening) for each Gaussian component fitted to [Fe II], $\mathrm{Pa} \beta$, and $\mathrm{H}_{2}$ emission lines of every galaxy in our sample. The name of the galaxy is indicated at the upper left-hand corner and the vertical bars on the right indicate which component the maps are referring to: green to the narrow and purple to the broad components; in the case of Mrk 607, which has three components, green corresponds to the narrow/disc, blue and red to the blueshifted and redshifted components, respectively. The grey regions in these maps represent locations where the amplitude of the corresponding Gaussian component is lower than three times the standard deviation of the continuum level next to each emission line.

In Appendix A (shown as supplementary material), we present the emission line channel maps for the three emission lines fitted and analysed here. These channel maps support our interpretation of the molecular and ionized gas kinematics in the galaxies.

\subsubsection{Highlights from the kinematic maps}

A detailed discussion of all the maps, including the flux distributions, and a comparison with previous results for each galaxy are presented in the Appendix B.

Here we summarize the main features observed in the gas kinematics: (i) the narrow/single component is dominated by rotation, i.e. from gas orbiting in the disc, except the case of NGC 5899, for which it is due to ionized outflows; (ii) the broad component in the ionized gas is detected in three galaxies - NGC 5506, NGC 3227 , and NGC 5899 - and it is interpreted as tracing gas outflow; (iii) a broad component in the molecular gas is seen only in NGC 5899 interpreted as originating from the interaction between the ionized outflows and the molecular gas in the disc; (iv) an equatorial outflow is detected in Mrk 607; (v) the narrow component kinematics although dominated by rotation - usually shows deviation, which are mostly present in the ionized gas, but also in $\mathrm{H}_{2}$ in the case of NGC 3227.

\section{DISCUSSION}

\subsection{Rotation models}

In Figs 1-6, we observe that the narrow or single component of both molecular and ionized gas velocity fields shows a rotation pattern, but some deviations from pure rotation are also observed. The molecular gas is usually more restricted to the plane of the disc (e.g. Reunanen, Kotilainen \& Prieto 2002; Riffel et al. 2015), therefore it is prone to having a velocity field dominated by rotational motions. We then fit the narrow/single component velocity fields by a rotation disc model, to investigate the residual velocity maps (observed - model) and identify non-circular motions. The $\mathrm{H}_{2}$ velocity fields are modelled by the following equation:

$V(R, \psi)=V_{\mathrm{sys}}+\frac{A R \cos \left(\psi-\psi_{0}\right) \sin \theta \cos ^{p} \theta}{\left\{R^{2}\left[\sin ^{2}\left(\psi-\psi_{0}\right)+\cos ^{2}\left(\psi-\psi_{0}\right)\right]+C_{0} \cos ^{2} \theta\right\}^{p / 2}}$,

where $R$ is the distance of each spaxel to the nucleus, $V_{\text {sys }}$ is the systemic velocity of the galaxy, $A$ is the velocity amplitude, $\psi$ is the position angle of each spaxel in the plane of the sky, $\psi_{0}$ is the orientation of the line of the nodes, $\theta$ is the inclination of the disc, $C_{0}$ is a concentration parameter, and the parameter $p$ defines the slope of the rotation curve at the largest radii and is restricted to 1 $\leq p \leq 3 / 2$. The equation is based on Bertola et al. (1991) and has already been used in previous works by our group using similar data
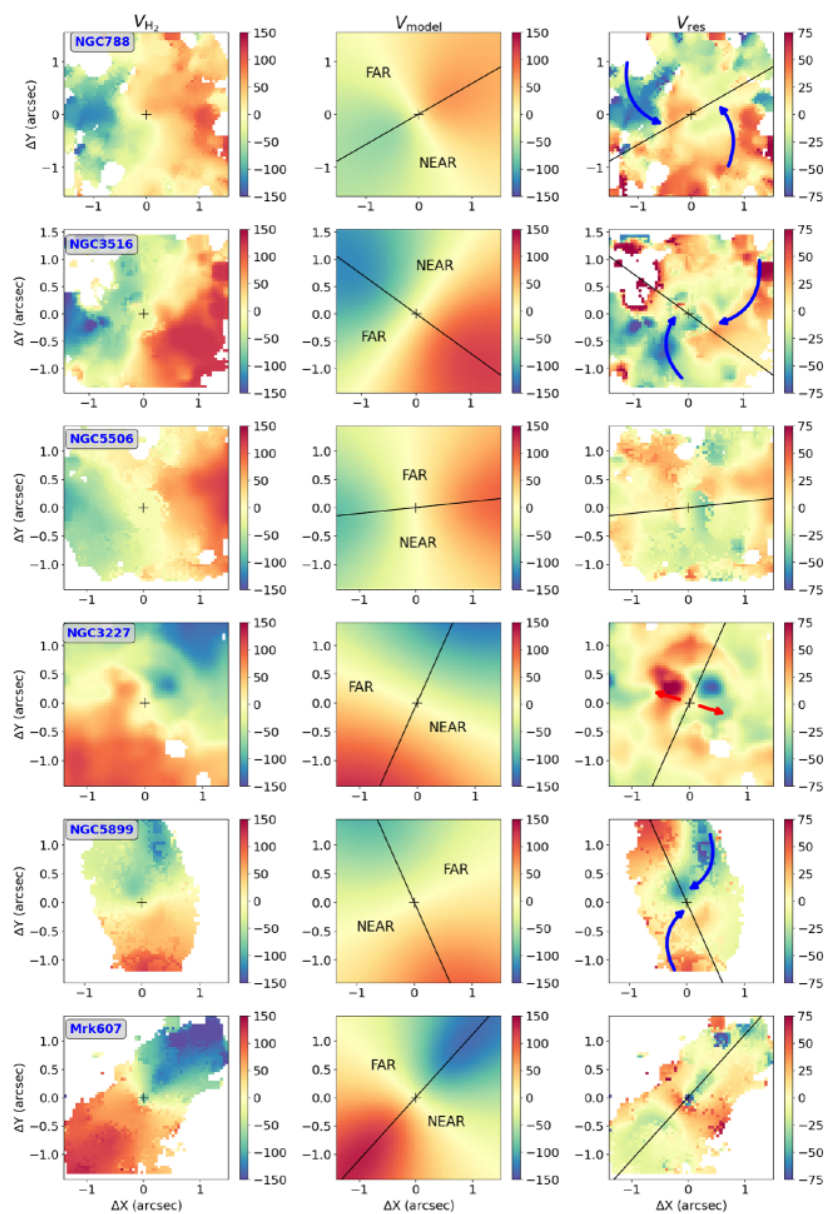

Figure 7. Molecular gas in the disc of the galaxies. The first column shows the $\mathrm{H}_{2}$ velocity field as in Figs $1-6$. The second column shows the rotation models. The black line indicates the orientation of the line of the nodes $\left(\psi_{0}\right)$ as derived by Riffel et al. (2017). The third column shows the residuals ( $V_{\text {res }}=$ $\left.V_{\mathrm{H}_{2}}-V_{\text {model }}\right)$. The black cross marks the position of the AGN. The red arrows indicate residuals interpreted as outflows and blue arrows the inflows.

(e.g. Schnorr-Müller et al. 2014; Diniz et al. 2015; Brum et al. 2017, 2019).

During the fit of the $\mathrm{H}_{2}$ velocity fields we fix $\psi_{0}$ and $\theta$ to the values obtained from previous modelling of the stellar velocity fields (Riffel et al. 2017). We adopt this procedure because the non-circular motions in the gas kinematics - e.g. inflows and outflows - will influence the fit possibly leading to a wrong rotation field, while the stars are a better tracer of circular motions dominated by the gravitational potential of the galaxies. In the case of Mrk 607, to take into account the counter-rotation of the gas (Riffel et al. 2017; Freitas et al. 2018), we subtract $180^{\circ}$ from $\psi_{0}$ and adopt this new value as the orientation of the line of the nodes. The kinematical centre is assumed as the position corresponding to the peak of the continuum flux in the $K$ band. We also assume $p=3 / 2$ that our previous studies have shown to reproduce better the inner regions. Previous assumptions of a flat rotation curve $(p=1)$ are better suited to the outer regions (beyond the inner kpc probed here) of disc galaxies. Therefore, $A$, $V_{\text {sys }}$, and $C_{0}$ are the only free parameters of the model. The middle column of Fig. 7 shows the modelled rotation velocity fields.

We use the colour maps from Martini et al. (2003a) to determine the far and near sides of the galaxies as indicated in the central panel of Fig. 7. The $V-H$ colour map allows the identification of the 
most obscured side of the galaxy, which can be identified with its near side. This is due to the fact that the light emitted by the central, most luminous region of the galaxy (bulge and surroundings), due to the galaxy inclination, becomes partially hidden by dust lanes in the near side of the disc, while in the far side this does not happen. Thus, the near side is redder and looks dustier than the far side the colour maps of the galaxies.

In NGC 3227, the dust lanes are not as clear as in the other galaxies, but our determination agrees with those from previous works (Barbosa et al. 2009; Alonso-Herrero et al. 2019). HST data in $V$ - and $H$-band images are not available for NGC 5899, therefore we use the Pan-STARRS image (Fig. 5), assuming the arms are trailing, and from the observed kinematics, determine the southwest as the near side of the galaxy. We use the galaxy major axis determined by Riffel et al. (2017) to separate the near from the far sides.

In order to identify non-circular motions in the $\mathrm{H}_{2}$ kinematics, we build the residual velocity maps $\left(V_{\text {res }}=V_{\mathrm{H}_{2}}-V_{\text {model }}\right)$ presented in the third column of Fig. 7. If the gas is located in the plane of the disc, blueshifts in the far side and redshifts in the near side indicate that the gas is moving towards the centre of the galaxy. If the opposite is observed, redshift in the far side and blueshifts in the near side, the residuals may be tracing outflows. In all cases, the residual velocities are much smaller than the observed velocity amplitudes, indicating that the velocity fields for the narrow component are dominated by rotation. However, systematic deviations from pure rotation are seen in all galaxies suggesting that besides rotation, the gas displays also non-circular motions. Thus, we summarize and interpret the observed velocity residuals for each galaxy as follows.

$N G C$ 788. Mostly blueshifts in the far side and redshifts in the near side of the galaxy with velocities of $\sim 50 \mathrm{~km} \mathrm{~s}^{-1}$. Assuming that the gas is in the disc, as indicated by the low $\sigma$ values $\left(\sim 60 \mathrm{~km} \mathrm{~s}^{-1}\right)$ observed (Fig. 1), the residual velocities are consistent with streaming motions towards the centre. On the other hand, another possible explanation, as the residuals are more spread over the FoV, is that these disturbances in the velocity field originate from the interaction between the possible [Fe II] bipolar outflow and the molecular gas in the disc.

$N G C$ 3516. The residual map shows mostly redshifts in the near side of the galaxy and blueshifts in its far side. The enhanced $\mathrm{H}_{2}$ flux values outside the nucleus are cospatial with the highest residuals and with the lowest $\mathrm{H}_{2} \sigma$ values (Fig. 2). The residual maps, $\mathrm{H}_{2}$ flux distribution, and velocity dispersion support the presence of streaming motions towards the centre of this galaxy.

NGC 5506. Some redshifts are observed in the far side and blueshifts in the near side of the galaxy, approximately along the AGN ionization axis at position angle of $22^{\circ}$ (Fischer et al. 2013). This result is consistent with the orientation of the ionized outflow observed in $\mathrm{Pa} \beta$ and [Fe II] (see Section $\mathrm{B} 3$ ). The $\mathrm{H}_{2}$ residuals may be tracing the emission of molecular gas located in the outer layers of the outflow seen in the ionized gas.

$N G C$ 3227. In the central 0.7 arcsec, redshifts are observed in the far side and blueshifts in the near side of the galaxy, indicating the presence of a compact outflow with velocity amplitudes $\sim 80 \mathrm{~km} \mathrm{~s}^{-1}$. Davies et al. (2014) do not see the molecular outflow directly, instead interpret the $\mathrm{H}_{2}$ velocity field distortions as due to an interaction between the ionized outflow and the gas in the disc. On the other hand, a compact molecular outflow seen in the $\mathrm{CO}(3-2)$ along the galaxy minor axis with an extension of $70 \mathrm{pc}$ has been detected for the cold gas (Alonso-Herrero et al. 2019), supporting our interpretation. The outflow structure for this galaxy is probably a combination of the scenarios proposed by Fischer et al. (2013), where we see inside the ionized outflow bicone (Fig. 4), and by Alonso-Herrero et al. (2019), where the molecular emission is more compact and clumpy.

$N G C$ 5899. This galaxy shows the clearest signatures of gas inflows in our sample. A strip of blueshifted gas $\left(\sim 80 \mathrm{~km} \mathrm{~s}^{-1}\right)$ is observed in the north-west side of the nucleus (the far side of the galaxy). Redshifts with similar velocities are seen on the near side of the disc. Both, blueshifts and redshifts, are seen in regions with low $\mathrm{H}_{2}$ velocity dispersion (Fig. 5), indicating that the gas is located in the plane of the galaxy and thus consistent with inflows.

Mrk 607. We use the single Gaussian fit to obtain the rotation model for this galaxy as it represents the bulk of the emission from the disc. The residuals are small at most locations. The residual map presents an excess of redshifts perpendicular to $\psi_{0}$, which may be associated with the equatorial outflows observed for this galaxy (see Freitas et al. 2018, and discussion below).

\subsection{Inflow properties}

The feeding to the AGN can be measured through kinematic signatures on the velocity maps that indicate the gas is flowing towards the centre. In $\mathrm{H}_{2} 2.1218 \mu \mathrm{m}$ such signatures have been observed in some nearby active galaxies, including a few galaxies of the AGNIFS sample (e.g. Riffel et al. 2008, 2013b; Hicks et al. 2009; Müller Sánchez et al. 2009; Diniz et al. 2015). In Section 4.1, we present the $\mathrm{H}_{2}$ residual velocity maps for the six galaxies and all of them present disturbances. For three galaxies, NGC 788, NGC 3516, and NGC 5899, a possible interpretation for the residuals - excess of blueshifts in the near side and of redshifts in the far side - is the presence of the gas inflows. Although we cannot rule out some other effect, such as disturbances due to ionized outflows, we adopt here the inflow hypothesis.

Based on Scoville et al. (1982) we can estimate the mass of hot molecular gas in the disc component gas from the following equation:

$$
\left(\frac{M_{\mathrm{H}_{2}}}{\mathrm{M}_{\odot}}\right)=5.0776 \times 10^{13}\left(\frac{F_{\mathrm{H}_{2} 2.1218}}{\mathrm{erg} \mathrm{s}^{-1} \mathrm{~cm}^{-2}}\right)\left(\frac{D}{\mathrm{Mpc}}\right)^{2},
$$

where $F_{\mathrm{H}_{2} 2.1218}$ flux of the narrow component of $\mathrm{H}_{2} 2.1218 \mu \mathrm{m}$ summed over the whole FoV and $D$ is the redshift distance of the galaxy. This equation is valid for gas in local thermodynamic equilibrium $\left(T_{\mathrm{vib}} \approx 2000 \mathrm{~K}\right)$ that is consistent with the temperatures we measure for these galaxies (Riffel et al. 2021b).

The equation above provides the total mass of hot molecular gas in the disc (rotating gas+inflows) that is extended over a region with a radius $r_{\mathrm{d}} \approx 1$ arcsec in these three galaxies. This radius encloses an area smaller than the NIFS FoV and is chosen because usually the borders present poorer quality in the emission line fit. The inflowing structures (see $V_{\text {res }}$ in Fig. 7) appear in pairs with velocities of opposite values in each side of the galaxy resembling double spiral arms $\left(n_{\text {arms }}=2\right)$ that are also clear in the large-scale image of NGC 5899 (Fig. 5) where they are more collimated, each with a radius $r \approx 0.3$ arcsec and each with a velocity of $v_{\mathrm{obs}}=$ $80 \mathrm{~km} \mathrm{~s}^{-1}$. In NGC 788 and NGC 3516, the spiral arms are not seen in their large-scale images but are observed in the velocity residual maps $\left(v_{\mathrm{obs}}=50 \mathrm{~km} \mathrm{~s}^{-1}\right)$, being less collimated than for NGC 5899, with a radius $r \approx 0.5$ arcsec. The corresponding physical radius for $r_{\mathrm{d}}$ and $r$ are presented in Table 1 .

To obtain the mass inflow rate we assume the gas is moving towards the centre over a circular cross-section with radius $r$ in a spiral structure with two arms $\left(n_{\text {arms }}=2\right)$ provided by the following 
Table 1. Inflow properties: (1) Galaxy name; physical scale, in parsec, of the (2) $\mathrm{H}_{2}$ disc radius and of the (3) cross-section radius; (4) velocity of the inflowing gas in $\mathrm{km} \mathrm{s}^{-1}$; and (5) mass inflow rate in $10^{-3} \mathrm{M}_{\odot} \mathrm{yr}^{-1}$.

\begin{tabular}{lcccc}
\hline $\begin{array}{l}\text { Galaxy } \\
(1)\end{array}$ & $r_{\mathrm{d}}$ & $r$ & $v_{\text {in }}$ & $\dot{M}_{\mathrm{H}_{2}}$ \\
$(2)$ & $(3)$ & $(4)$ & $(5)$ \\
\hline NGC 788 & 278 & 139 & 140.8 & 0.9 \\
NGC 3516 & 181 & 91 & 160.1 & 0.62 \\
NGC 5899 & 176 & 53 & 90 & 0.1 \\
\hline
\end{tabular}

equation:

$\dot{M}_{\mathrm{H}_{2}}=2 m_{\mathrm{p}} N_{\mathrm{H}_{2}} v_{\text {in }} \pi r^{2} n_{\text {arms }}$,

where $m_{\mathrm{p}}$ is the proton mass and the inflow velocity $v_{\text {in }}=v_{\text {obs }} / \sin i$, and $v_{\text {obs }}$ is the observed velocity inferred from the velocity residual maps (50 km s${ }^{-1}$ for NGC 788 and NGC 3516 and $80 \mathrm{~km} \mathrm{~s}^{-1}$ for NGC 5899), corrected by the inclination of the disc $i$ obtained from the modelling of the stellar velocity field by Riffel et al. (2017). The other term of this equation is $N_{\mathrm{H}_{2}}$, the molecular hydrogen density, obtained through $N_{\mathrm{H}_{2}}=\frac{M_{\mathrm{H}_{2}}}{2 m_{\mathrm{p}} \pi r_{\mathrm{d}}^{2} h} . M_{\mathrm{H}_{2}}$ is the molecular gas mass as calculated by equation (1) that is distributed in a thick disc with radius $r_{\mathrm{d}}$ with a height $h$ adopted as the typical value of $30 \mathrm{pc}$ (Hicks et al. 2009).

The masses of hot molecular gas range from 177 to $377 \mathrm{M}_{\odot}$ and, as expected, agree with the values we have already calculated for these galaxies (Schönell et al. 2019). The densities, in the range 1.05$1.49 \times 10^{-3} \mathrm{~cm}^{-3}$, are systematically lower than we have previously measured in Mrk 79 and in NGC 2110: $3.3 \times 10^{-3} \mathrm{~cm}^{-3}$ (Riffel et al. 2013b) and $6.22 \times 10^{-3} \mathrm{~cm}^{-3}$ (Diniz et al. 2015), respectively. Although we find this discrepancy the values have the same order of magnitude and can be considered consistent with each other. In Table 1, the inflow properties are summarized. The inflow velocities are higher in NGC 3516 reaching $160 \mathrm{~km} \mathrm{~s}^{-1}$. Mass inflow rates range from 0.1 to $0.9 \times 10^{-3} \mathrm{M}_{\odot} \mathrm{yr}^{-1}$ and are similar to values obtained for similar objects in the hot molecular gas (Riffel et al. 2013b; Diniz et al. 2015; Storchi-Bergmann \& Schnorr-Müller 2019).

\subsection{Outflows properties}

As mentioned in Section 3.2.1, in NGC 5506, NGC 3227, and NGC 5899 the ionized gas shows signatures of outflows, traced by the broad component, while in Mrk 607 an equatorial outflow is observed. The major part of this section is dedicated to estimate the mass, mass outflow rate, and kinetic power of the ionized outflows.

We also observe molecular outflows in NGC 5899 and in Mrk 607, traced by the distinct kinematic components. In the residual velocity maps (Section 4.1) evidence for the presence of molecular outflows is observed in NGC 5506 and NGC 3227. The hot molecular hydrogen represents a small fraction of the total molecular gas of the galaxy, thus we expect the mass outflow rates and powers to be small compared to the more powerful ionized outflows. The derived quantities will be presented and discussed in Section 4.3.4.

\subsubsection{Fraction of the gas in the outflows}

We assume the outflow is traced only by the broad component in NGC 5506 and NGC 3227. Thus, all the calculations performed in this section are based on this component. In NGC 5899, both components fitted to the ionized gas emission lines are produced by a bipolar/biconical outflow and in Mrk 607 the blueshifted and redshifted components are describing an equatorial outflow, thus we will adopt the flux of the outflowing gas as the sum of those of these two components.

The mass of $\mathrm{H}^{+}$in the outflow is estimated using the same equation presented in Storchi-Bergmann et al. (2009), but instead of the flux of the $\operatorname{Br} \gamma\left(F_{\mathrm{Br} \gamma}\right)$ we use the theoretical ratio between $\operatorname{Pa} \beta$ and $\mathrm{Br} \gamma$ fluxes for the case $\mathrm{B}$ recombination as $\frac{F_{\mathrm{Pa} \beta}}{F_{\mathrm{Br} \gamma}}=5.88$, assuming an electron temperature of $10000 \mathrm{~K}$ and the low-density regime (Osterbrock \& Ferland 2006) to obtain it in terms of the $\mathrm{Pa} \beta$ flux, resulting in the following equation:

$$
\left(\frac{M_{\mathrm{HII}}}{\mathrm{M}_{\odot}}\right)=5.1 \times 10^{18}\left(\frac{F_{\mathrm{Pa} \beta}}{\mathrm{erg} \mathrm{cm}^{-2} \mathrm{~s}^{-1}}\right)\left(\frac{D}{\mathrm{Mpc}}\right)^{2}\left(\frac{n_{\mathrm{e}}}{\mathrm{cm}^{-3}}\right)^{-1},
$$

where $F_{\mathrm{Pa} \beta}$ is the broad component $\mathrm{Pa} \beta$ flux. We sum the fluxes over the spaxels within a radius of $100 \mathrm{pc}$ that corresponds to $\sim 1 \operatorname{arcsec}$ or less, in these galaxies (see the scale in the continuum maps of Figs 1-6) and encloses the outflow structure in theses galaxies. We choose this same physical radius $(100 \mathrm{pc})$ for the outflow to allow the comparison between the galaxies. The electron density is given by $n_{\mathrm{e}}$ and $D$ is the redshift distance of the galaxy.

Davies et al. (2020) studied different methods to estimate the gas electron density in active galaxies in optical wavelengths. The most traditional is the method based on the [S II] $\lambda \lambda 6716,6731$ emission lines that provides typical densities for the narrow line region (NLR) of $\sim 500 \mathrm{~cm}^{-3}$ (e.g. Dors et al. 2015; Freitas et al. 2018; Kakkad et al. 2018). The method based on auroral [O II] $\lambda \lambda 7320$, 7331 and transauroral [S II] $\lambda \lambda 4069,4076$ lines provides densities $\sim 2000 \mathrm{~cm}^{-3}$, but its use is limited by the usual weakness of these emission lines that are hard to detect. The third method presented by the authors is based on the estimate of the ionization parameter (Baron \& Netzer 2019) and systematically provides higher densities compared to those based on the [S II] doublet, reaching up to $22000 \mathrm{~cm}^{-3}$. High densities $\left(10^{4} \mathrm{~cm}^{-3}\right)$ are also observed using the $H$-band [Fe II] (Storchi-Bergmann et al. 2009; Riffel 2021) and optical [Ar IV] (Riffel et al. 2021a) lines. Our $J$ - and $K$-band spectra do not include emission lines that can be used to determine the density of the outflows and to estimate the mass of the outflowing gas we have to assume a density value. We follow the same procedure adopted by Kakkad et al. (2020) where two densities are used: one low $\left(500 \mathrm{~cm}^{-3}\right)$ and one high $\left(10000 \mathrm{~cm}^{-3}\right)$.

The outflow masses calculated inside the $100 \mathrm{pc}$ radius are presented in Table 2 where $M_{\mathrm{H} \text { II }}^{\mathrm{ld}}$ and $M_{\mathrm{H} \text { II }}^{\mathrm{hd}}$ are the ionized hydrogen masses assuming 500 and $10000 \mathrm{~cm}^{-3}$ densities, respectively. The masses estimates for the low-density regime are 20 times higher than the masses estimated assuming the higher density (which is the ratio between the gas densities).

For NGC 5899, both components seen in the ionized gas emission lines are consistent with a bipolar outflow and the outflow dominates the emission within $100 \mathrm{pc}$. For the other galaxies, we can compare the masses of the gas in the outflow with the total mass of ionized gas within $100 \mathrm{pc}$, by computing the mass of gas in the disc using the fluxes of the narrow components. We find that the outflows correspond to fractions of 84 per cent, 46 per cent, and 42 per cent for NGC 3227, NGC 5506, and Mrk 607, respectively.

\subsubsection{Ionized gas mass outflow rates}

The geometry of the outflow impacts the way we estimate its rate and kinetic power (Lutz et al. 2020). Assuming the gas in the outflow is uniformly distributed inside a biconical or spherical geometry the mass outflow rate is given by (e.g. Harrison et al. 2014; Fiore et al. 
Table 2. Outflow properties: (1) Galaxy name; masses of ionized gas calculated for (2) low density (500 $\left.\mathrm{cm}^{-3}\right)$ and (3) high density $\left(10000 \mathrm{~cm}^{-3}\right)$ in units of $10^{4} \mathrm{M}_{\odot}$; we assume spherical [columns (4)-(6)] and biconical geometries [columns (7)-(9)] with the outflow velocity is in units of $\mathrm{km} \mathrm{s}^{-1}$, and the mass outflow rates in $\mathrm{M}_{\odot} \mathrm{yr}^{-1}$; (10) velocity dispersion of the outflow in units of km s${ }^{-1}$; (11) kinetic power, in units of $10^{40} \mathrm{erg} \mathrm{s}^{-1}$, calculated for the mass outflow rate in column (8), except in Mrk 607 where the outflow is spherical and we use the value in column (5); (12) kinetic efficiency of the outflows in percentage.

\begin{tabular}{lccccccccccc}
\hline Galaxy & $M_{\mathrm{H} \text { II }}^{\text {ld }}$ & $M_{\mathrm{H} \text { II }}^{\text {hd }}$ & $\begin{array}{c}v_{\text {out }} \\
(1)\end{array}$ & $\begin{array}{c}\text { Sphere } \\
\dot{M}_{\text {out }}^{\text {ld }}\end{array}$ & $\begin{array}{c}\dot{M}_{\text {out }}^{\text {hd }} \\
(5)\end{array}$ & $\begin{array}{c}v_{\text {out }} \\
(7)\end{array}$ & $\begin{array}{c}\text { Bicone } \\
\dot{M}_{\text {out }}^{\text {ld }}\end{array}$ & $\begin{array}{c}\dot{M}_{\text {out }}^{\text {hd }} \\
(8)\end{array}$ & $\begin{array}{c}\sigma_{\text {out }} \\
(9)\end{array}$ & $\begin{array}{c}\dot{E}_{\text {kin }} \\
(11)\end{array}$ & $\begin{array}{c}\dot{E}_{\text {kin }} \\
(12)\end{array}$ \\
\hline NGC 5506 & 70.90 & 3.54 & 100 & 2.17 & 0.11 & 576 & 12.49 & 0.62 & 350 & 275 & 0.71 \\
NGC 3227 & 3.72 & 0.19 & 150 & 0.17 & 0.008 & 155 & 0.18 & 0.008 & 250 & 1.18 & 0.02 \\
NGC 5899 & 4.01 & 0.20 & 100 & 0.12 & 0.006 & 110 & 0.13 & 0.007 & 300 & 1.20 & 0.03 \\
Mrk 607 & 3.55 & 0.18 & 100 & 0.108 & 0.005 & - & - & - & 100 & 0.13 & 0.005 \\
\hline
\end{tabular}

2017; Kakkad et al. 2020)

$\dot{M}_{\text {out }}=3 \frac{M_{\text {out }} v_{\text {out }}}{R_{\text {out }}}$,

where $M_{\text {out }}$ is the mass of the gas that is outflowing, $v_{\text {out }}$ is outflow velocity, and $R_{\text {out }}$ its radius. We consider $M_{\text {out }}=M_{\mathrm{H} \mathrm{II}}$ shown in Table 2 and calculated within $R_{\text {out }}=100$ pc. If we assume a biconical geometry, the outflow velocity $\left(v_{\text {out }}\right)$ is given by $\frac{v_{\text {obs }}}{\sin \theta}$ with $v_{\text {obs }}$, the observed velocity, inferred from the velocity fields and $\theta$ the inclination of the bicone with respect to the plane of the sky (see discussion below). On the other hand, if we assume the outflow is spherical, we simply have $v_{\text {out }}=v_{\text {obs }}$. The observed velocity, fourth column of Table 2 , is inferred from the $\mathrm{Pa} \beta$ velocity fields of the components that represent the outflow (see the discussion above).

Fischer et al. (2013) used long slit HST-Space Telescope Imaging Spectrograph (STIS) data to observe and model the [O III] $\lambda 5007$ as a biconical outflow in nearby galaxies, including NGC 3227 and NGC 5006. In NGC 3227 the outflow is almost perpendicular to the plane of the sky, $\left(\theta=75^{\circ}\right)$ in agreement with our broad component velocity fields (see Fig. 4), even though we do not observe the back part of the outflow as predicted by their model. For NGC 5506 the biconical structure is not so clear in our data (see Fig. 3), but the redshifts to the south-east of the nucleus contrasting with the blueshifts seen in the rest of the FoV in the [Fe II] velocity field support its presence. In this case, the ionization axis has a small inclination, $\theta=10^{\circ}$ (Fischer et al. 2013). For Mrk 607, from the blue and red components flux distributions and velocity fields (Fig. 6), we infer the outflow has a spherical symmetry.

The outflow in NGC 5899 is not modelled by Fischer et al. (2013) and in Ruschel-Dutra et al. (2021) an expanding spherical shape is assumed to reproduce the outflow and thus an inclination is not estimated. But the biconical structure is clear in the velocity fields of $\mathrm{Pa} \beta$ and $[\mathrm{Fe} \mathrm{II}]$ of NGC 5899. The zero velocity regions (see the central maps for both components of [Fe II] and $\mathrm{Pa} \beta$ in Fig. 5) trace a wall of the bicone parallel to the plane of the sky with the blueshifts and redshifts tracing the front and back walls, respectively. With this geometry in mind, we assume the inclination of the bicone is equal to the apparent opening angle, measured directly from the velocity fields, resulting in $\theta=65^{\circ}$.

In Table 2, we present the velocities and mass outflow rates for spherical (columns 4-6) and biconical (columns 7-9) geometries. The two different mass outflow rates for each geometry are obtained considering the low-density (superscript ld) and high-density (superscript hd) regimes (columns 2 and 3). In the case of Mrk 607, the outflow is spherical, and we thus do not estimate its value for the biconical geometry. Comparing the mass outflow rates for the two geometries we see that due to the high inclination assumed for
NGC 3227 both rates result similar and we can thus infer the outflow is well represented by a spherical one. NGC 5899 also does not have a big discrepancy between the two mass outflow rates. The biggest difference is seen for NGC 5506, where the bicone axis is almost perpendicular to the plane of the sky leading to a high corrected velocity that leads to $\dot{M}_{\text {out }}^{\text {ld }}=12.5 \mathrm{M}_{\odot} \mathrm{yr}^{-1}$ when we take into account this geometry.

\subsubsection{Kinetic power}

We can use the mass outflow rates derived in the previous section to estimate the power of the outflows by

$\dot{E}_{\text {kin }} \approx \frac{\dot{M}_{\text {out }}}{2}\left(v_{\text {out }}^{2}+3 \sigma_{\text {out }}^{2}\right)$.

The term associated with $\sigma_{\text {out }}^{2}$ is related with the disordered motions of the gas, which are significative in a gas in outflow. We adopt the lowest density and highest velocity regimes to assume $\dot{M}_{\text {out }}$ as the value given in column (8) of Table 2 and $v_{\text {out }}=v_{\text {obs }} / \sin \theta$. The velocity dispersion of the outflow, $\sigma_{\text {out }}$, is estimated from the $\mathrm{Pa} \beta$ broad component in NGC 5506 and NGC 3227, for the galaxies NGC 5899 and Mrk 607 we assume $\sigma_{\text {out }}$ is the highest value from any of the components that represent the outflow. These values, as well as the $\dot{E}_{\text {kin }}$, are presented in Table 2 .

\subsubsection{Molecular gas outflows}

In this section, we determine the mass, mass outflow rate, and power of the molecular outflows observed in the galaxies. In NGC 5899 and Mrk 607 the outflowing gas is assumed to be described by the broad component of the emission line. The mass of the gas in the outflow can be determined by equation (1), where $F_{\mathrm{H}_{2} 2.1218}$ is the flux of the broad component of the $\mathrm{H}_{2}$ emission line. We determine 35 and $26 \mathrm{M}_{\odot}$ as the masses of the $\mathrm{H}_{2}$ in the outflow for NGC 5899 and Mrk 607, respectively.

A rough estimate of the mass outflow rate of the molecular gas can be obtained by $\dot{M}_{\text {out }}^{\mathrm{H}_{2}}=\dot{M}_{\text {out }} \frac{M_{\mathrm{H}_{2}}}{M_{\text {out }}}$, where $M_{\text {out }}$ and $\dot{M}_{\text {out }}$ are the mass and outflow rate of the ionized gas outflow determined in Section 4.3.2. Thus, we determine the outflow rates of molecular gas being $1 \times 10^{-4}$ and $7.8 \times 10^{-5} \mathrm{M}_{\odot} \mathrm{yr}^{-1}$. For NGC 5899, where the ionized gas seems to be distributed in a bicone, we find no difference in the mass outflow rate when taking into account this particular geometry.

In the velocity residual maps (see Fig. 7 and Section 4.1) we observe residuals compatible with outflows in NGC 5506 and NGC 3227. Unlike the previous case, an isolated component is not describing this gas in the outflow, thus a different approach is 
necessary. We follow the procedure described in Diniz et al. (2015) where a compact molecular outflow was observed in the residual velocity map for NGC 2110. In NGC 5506, signatures of an outflow weak outflow are observed and possibly associated with the outer layers of the more powerful ionized gas outflow (see the third panel in the third column of Fig. 7), while in NGC 3227 the structure is more compact, but the similar velocity intensities in each side make the assumption of a biconical geometry plausible. Using equation (4) and assuming the biconical geometry, the molecular mass outflow rate is given by

$\dot{M}_{\text {out }}^{\mathrm{H}_{2}}=2 m_{\mathrm{p}} N_{\mathrm{H}_{2}} n v_{\text {out }} \pi r_{\mathrm{b}}^{2}$,

where $m_{\mathrm{p}}$ is the proton mass, $v_{\text {out }}$ is the outflow velocity, $r_{\mathrm{b}}$ is the cross-section radius of the bicone, 1 arcsec for NGC 5506 and $0.5 \operatorname{arcsec}$ for NGC 3227 , and $N_{\mathrm{H}_{2}}$ is the molecular hydrogen density estimated following the equation and assumptions in Section 4.2. We calculate the density for a gas located in a disc with a radius $r_{\mathrm{d}}=1$ arcsec and a height $h=30 \mathrm{pc}$, resulting in $7 \times 10^{-3}$ and $1.6 \times 10^{-2} \mathrm{~cm}^{-3}$ for NGC 5506 and NGC 3227, respectively.

We use $n=2$ to account for both sides of the bicone. The observed velocity of the outflow is corrected by the inclination of the bicone with respect to the plane of the sky: $\theta=10^{\circ}$ for NGC 5506 and $\theta=75^{\circ}$ for NGC 3227 (Fischer et al. 2013). Thus, we calculate for NGC 5506: $v_{\text {out }}=143 \mathrm{~km} \mathrm{~s}^{-1}$ and $\dot{M}_{\text {out }}^{\mathrm{H}_{2}}=5.5 \times 10^{-3} \mathrm{M}_{\odot} \mathrm{yr}^{-1}$; and for NGC 3227: $v_{\text {out }}=77 \mathrm{~km} \mathrm{~s}^{-1}$ and $\dot{M}_{\text {out }}^{\mathrm{H}_{2}}=6.4 \times 10^{-4} \mathrm{M}_{\odot} \mathrm{yr}^{-1}$. The kinetic power of the hot molecular outflows ranges from $10^{35}$ to $10^{38} \mathrm{erg} \mathrm{s}^{-1}$, being four orders of magnitude smaller than the powers estimated for the outflows of ionized gas.

\section{THE IMPACT OF INFLOWS AND OUTFLOWS ON THE GALAXIES}

In order understand the impact of the observed inflows and outflows on the host galaxies, it is necessary to estimate the bolometric luminosity, $L_{\mathrm{bol}}$, of these objects. For the galaxies in our sample we can use the known Swift-BAT luminosity $\left(L_{\mathrm{X}}\right)$ to estimate $L_{\mathrm{bol}}$ using $\log L_{\mathrm{bol}}=0.0378\left(\log L_{\mathrm{X}}\right)^{2}-2.03 \log L_{\mathrm{X}}+61.6$ (Ichikawa et al. 2017).

First, we can check if the inflows observed and quantified in NGC 788, NGC 3516, and NGC 5899 can feed the AGN in each of these galaxies. In order to do so, we estimate the mass accretion rate by

$\dot{m}=\frac{L_{\text {bol }}}{c^{2} \eta}$,

where is $c$ the light speed and $\eta$ is the rest-frame mass conversion efficiency factor adopted as $\eta=0.1$ (Frank, King \& Raine 2002). We find $0.007 \leq \dot{m}\left(\mathrm{M}_{\odot} \mathrm{yr}^{-1}\right) \leq 0.12$ that is higher than the mass inflow rate of hot molecular gas for any of these galaxies. This indicates that the inflows in hot molecular gas alone we observe are not enough to power the AGN and maintain their activity at the current luminosity. However, the hot molecular gas corresponds only to a small fraction of the total amount of gas in the inner region of galaxies, with masses of cold gas being $10^{5}-10^{7}$ times larger than that in the hot molecular phase (e.g. Dale et al. 2005; Müller Sánchez et al. 2006; Mazzalay et al. 2013).

The comparison between the kinetic power of the outflows and the bolometric luminosity, usually referred as the kinetic efficiency, is presented in the last column of Table 2 for the outflows of ionized gas. Hopkins \& Elvis (2010) found that for a hot gas outflow to be efficient in blowing the cold gas supply of the galaxy hosting an AGN its power needs to be, at least, 0.5 per cent of the galaxy's bolometric luminosity
$\left(L_{\mathrm{bol}}\right)$, which, in a direct comparison with our measurements, is only reached by NGC 5506. However, Harrison et al. (2018) point out that the observed kinetic efficiencies, obtained from the gas kinematics, should not be directly compared to theoretical simulations. The observed kinetic efficiencies account only for the mechanical effect of the outflow seen in an specific gas phase and not the initial AGN energy input to the galaxy as usually presented in the models.

We can also compare the mass accretion rate with mass outflow rate in NGC 5506, NGC 3227, NGC 5899, and Mrk 607. Specially NGC 5506, the galaxy with the highest mass outflow rates (0.11$12.49 \mathrm{M}_{\odot} \mathrm{yr}^{-1}$ considering the different densities and geometries), has $\dot{m}=0.067 \mathrm{M}_{\odot} \mathrm{yr}^{-1}$ that indicates more gas is disturbed and flowing out of the central region than it is directed to the feeding of the SMBH. This result agrees with the kinetic efficiency of 0.7 per cent we calculated previously. The only galaxy where we simultaneously observe inflows and outflows is NGC 5899, which shows an outflow rate in ionized gas about one order of magnitude larger than the inflow rate in hot molecular gas.

The molecular outflows we observe in the galaxies have a very low kinetic efficiency $\left(1.4 \times 10^{-6}-3.2 \times 10^{-5}\right.$ per cent), but, as stated before, the hot molecular gas represents a small fraction of the total mass of molecular gas and the kinetic efficiency does not account for all the impact an outflow can have on the host. Also, many assumptions are made in order to estimate the rates and powers and we understand they are highly uncertain. Nevertheless, we do observe disturbed molecular gas in four of our galaxies, indicating it not always displays 'well-behaved' motions in the stellar disc.

Thus, we find that the inflow rates in hot molecular gas in our sample are not enough to power their AGN activity at the current accretion rates (cold molecular gas may dominate the SMBH feeding processes) and the ionized outflows may only be able to redistribute the gas in the central kpc, which will be still available for future star formation.

\section{CONCLUSIONS}

We studied six nearby AGN hosts, from the AGNIFS selection of nearby active galaxies, namely NGC 788, NGC 3516, NGC 5506, NGC 3227, NGC 5899, and Mrk 607 using the Gemini NIFS integral field spectra in the $J$ and $K$ bands. For these galaxies we obtained the resolved ionized ([Fe II $] 1.2570 \mu \mathrm{m}$ and $\mathrm{Pa} \beta$ ) and molecular $\left(\mathrm{H}_{2} 2.1218 \mu \mathrm{m}\right)$ gas distributions and kinematics in their inner kiloparsec, and investigated the AGN feeding and feedback processes using spatially resolved observations of inflows and outflows. Bellow, we summarize the main conclusions drawn from this work.

(i) A multi-Gaussian fitting approach was adopted to describe the emission line profiles. The narrow Gaussian component traces the gas located in a disc that is dominated by a rotation pattern, except the ionized gas in NGC 5899 that shows all of its emission coming from the outflowing gas. The broad components - and in one case the double red and blueshifted components - trace non-circular motions.

(ii) A clear molecular outflow, traced by a broad component, is observed only for NGC 5899. It is probably originated from the interaction between the ionized outflow and the molecular gas in the disc.

(iii) Ionized gas outflows - traced by the broad or double components - are observed in four galaxies: NGC 5506, NGC 3227, NGC 5899, and Mrk 607. Molecular gas outflows are detected in NGC 5899, interpreted as due to the interaction between the ionized outflow and the gas in the disc, and in Mrk 607, interpreted as due to an equatorial outflow. 
(iv) The molecular gas velocity field is usually well described by a rotating disc model, but the velocity residuals indicate the presence of gas inflows in three galaxies, NGC 788, NGC 3516, and NGC 5899, and gas outflows in two, NGC 5506 and NGC 3227. Signatures of an equatorial outflow are observed in Mrk 607.

(v) The mass outflow rates of ionized gas in the four galaxies are in the range of $10^{-3}-10^{2} \mathrm{M}_{\odot} \mathrm{yr}^{-1}$. For the molecular gas outflows we measure mass outflows rates in the range $10^{-5}-10^{-3} \mathrm{M}_{\odot} \mathrm{yr}^{-1}$.

(vi) The hot molecular inflows cannot power and maintain the AGN at their current luminosity, as the mass inflow rates are three orders of magnitude lower than the mass accretion rates. This can be understood as due to the fact that the hot molecular gas traces only the 'hot skin' of a much larger molecular gas reservoir.

\section{ACKNOWLEDGEMENTS}

We thank an anonymous referee for the suggestions that helped us to improve this paper. MB thanks the financial support from Coordenação de Aperfeiçoamento de Pessoal de Nível Superior - Brasil (CAPES) - Finance Code 001. RAR acknowledges the support from Conselho Nacional de Desenvolvimento Científico e Tecnológico and Fundação de Amparo à Pesquisa do Estado do Rio Grande do Sul. RR thanks Conselho Nacional de Desenvolvimento Científico e Tecnológico (CNPq, Proj. 311223/20206, 304927/2017-1, and 400352/2016-8), Fundação de Amparo à Pesquisa do Estado do Rio Grande do Sul (FAPERGS, Proj. 16/25510000251-7 and 19/1750-2), and Coordenação de Aperfeiçoamento de Pessoal de Nível Superior (CAPES, Proj. 0001). NZD acknowledges partial support from FONDECYT through project 3190769. This study is based on observations obtained at the Gemini Observatory, which is operated by the Association of Universities for Research in Astronomy, Inc., under a cooperative agreement with the NSF on behalf of the Gemini partnership: the National Science Foundation (USA), National Research Council Canada (Canada), CONICYT (Chile), Ministerio de Ciencia, Tecnología e Innovación Productiva (Argentina), Ministério da Ciência, Tecnologia e Inovação (Brazil), and Korea Astronomy and Space Science Institute (Republic of Korea). This research has made use of NASA's Astrophysics Data System Bibliographic Services. This research has made use of the NASA/IPAC Extragalactic Database (NED), which is operated by the Jet Propulsion Laboratory, California Institute of Technology, under contract with the National Aeronautics and Space Administration.

\section{DATA AVAILABILITY}

The data used in this work are publicly available online via the GEMINI archive https://archive.gemini.edu/searchform, under the following program codes: GN-2012B-Q-45, GN-2013A-Q-48, GN2015A-Q-3, GN-2015B-Q-29, and GN-2016A-Q-6. The processed data cubes used in this paper will be shared on reasonable request to the corresponding author.

\section{REFERENCES}

Alonso-Herrero A. et al., 2019, A\&A, 628, A65

Arp H., 1966, ApJS, 14, 1

Barbosa F. K. B., Storchi-Bergmann T., Cid Fernandes R., Winge C., Schmitt H., 2006, MNRAS, 371, 170

Barbosa F. K. B., Storchi-Bergmann T., Cid Fernandes R., Winge C., Schmitt H., 2009, MNRAS, 396, 2

Barbosa F. K. B., Storchi-Bergmann T., McGregor P., Vale T. B., Rogemar Riffel A., 2014, MNRAS, 445, 2353
Baron D., Netzer H., 2019, MNRAS, 486, 4290

Benson A. J., Bower R. G., Frenk C. S., Lacey C. G., Baugh C. M., Cole S., 2003, ApJ, 599, 38

Bertola F., Bettoni D., Danziger J., Sadler E., Sparke L., de Zeeuw T., 1991, ApJ, 373, 369

Bieri R., Dubois Y., Silk J., Mamon G. A., Gaibler V., 2016, MNRAS, 455, 4166

Bower R. G., Benson A. J., Malbon R., Helly J. C., Frenk C. S., Baugh C. M., Cole S., Lacey C. G., 2006, MNRAS, 370, 645

Brum C., Riffel R. A., Storchi-Bergmann T., Robinson A., Schnorr Müller A., Lena D., 2017, MNRAS, 469, 3405

Brum C. et al., 2019, MNRAS, 486, 691

Caglar T. et al., 2020, A\&A, 634, A114

Chambers K. C. et al., 2019, preprint (arXiv:1612.05560)

Colbert E. J. M., Baum S. A., Gallimore J. F., O'Dea C. P., Christensen J. A., 1996, ApJ, 467, 551

Combes F. et al., 2013, A\&A, 558, A124

Costa T., Pakmor R., Springel V., 2020, MNRAS, 497, 5229

Crook A. C., Huchra J. P., Martimbeau N., Masters K. L., Jarrett T., Macri L. M., 2007, ApJ, 655, 790

Dale D. A., Sheth K., Helou G., Regan M. W., Hüttemeister S., 2005, AJ, 129,2197

Davies R. I. et al., 2006, ApJ, 646, 754

Davies R., Mark D., Sternberg A., 2012, A\&A, 537, A133

Davies R. I. et al., 2014, ApJ, 792, 101

Davies R. et al., 2020, MNRAS, 498, 4150

de Vaucouleurs G., de Vaucouleurs A., Corwin H. G., Jr, Buta R. J., Paturel G., Fouque P., 1991, Third Reference Catalogue of Bright Galaxies. Vol. I: Explanations and References. Vol. II: Data for Galaxies Between $0^{\mathrm{h}}$ and $12^{\mathrm{h}}$. Vol. III: Data for Galaxies Between $12^{\mathrm{h}}$ and $24^{\mathrm{h}}$. Springer, New York

Diniz M. R., Riffel R. A., Storchi-Bergmann T., Winge C., 2015, MNRAS, 453, 1727

Diniz S. I. F., Pastoriza M. G., Hernandez-Jimenez J. A., Riffel R., Ricci T. V., Steiner J. E., Riffel R. A., 2017, MNRAS, 470, 1703

Diniz M. R., Riffel R. A., Storchi-Bergmann T., Riffel R., 2019, MNRAS, 487, 3958

Dors O. L., Cardaci M. V., Hägele G. F., Rodrigues I., Grebel E. K., Pilyugin L. S., Freitas-Lemes P., Krabbe A. C., 2015, MNRAS, 453, 4102

El-Badry K., Wetzel A., Geha M., Hopkins P. F., Kereš D., Chan T. K., Faucher-Giguère C.-A., 2016, ApJ, 820, 131

Emonts B. H. C., Colina L., Piqueras-López J., Garcia-Burillo S., PereiraSantaella M., Arribas S., Labiano A., Alonso-Herrero A., 2017, A\&A, 607, A116

Fabian A. C., 2012, ARA\&A, 50, 455

Fathi K., Storchi-Bergmann T., Riffel R. A., Winge C., Axon D. J., Robinson A., Capetti A., Marconi A., 2006, ApJ, 641, L25

Ferrarese L., Merritt D., 2000, ApJ, 539, L9

Fiore F. et al., 2017, A\&A, 601, A143

Fischer T. C., Crenshaw D. M., Kraemer S. B., Schmitt H. R., 2013, ApJS, 209,1

Flewelling H. A. et al., 2020, ApJS, 251, 7

Frank J., King A., Raine D. J., 2002, Accretion Power in Astrophysics, 3rd edn. Cambridge Univ. Press, Cambridge

Freitas I. C. et al., 2018, MNRAS, 476, 2760

Galloway M. A. et al., 2015, MNRAS, 448, 3442

Garcia A. M., 1993, A\&AS, 100, 47

Garcia-Burillo S. et al., 2021, A\&A, 652, A98

Gaspari M., Ruszkowski M., Oh S. P., 2013, MNRAS, 432, 3401

Gebhardt K. et al., 2000, ApJ, 539, L13

Gnilka C. L. et al., 2020, ApJ, 893, 80

Goad J. W., Gallagher J. S., III, 1987, AJ, 94, 640

Granato G. L., De Zotti G., Silva L., Bressan A., Danese L., 2004, ApJ, 600, 580

Harrison C. M., 2017, Nat. Astron., 1, 0165

Harrison C. M., Alexander D. M., Mullaney J. R., Swinbank A. M., 2014, MNRAS, 441, 3306

Harrison C. M., Costa T., Tadhunter C. N., Flütsch A., Kakkad D., Perna M., Vietri G., 2018, Nat. Astron., 2, 198 
Hicks E. K. S., Davies R. I., Malkan M. A., Genzel R., Tacconi L. J., Müller Sánchez F., Sternberg A., 2009, ApJ, 696, 448

Hopkins P. F., 2012, MNRAS, 420, L8

Hopkins P. F., Elvis M., 2010, MNRAS, 401, 7

Hopkins P. F., Kocevski D. D., Bundy K., 2014, MNRAS, 445, 823

Ichikawa K., Ricci C., Ueda Y., Matsuoka K., Toba Y., Kawamuro T., Trakhtenbrot B., Koss M. J., 2017, ApJ, 835, 74

Ilić D. et al., 2020, A\&A, 638, A13

Ishibashi W., Fabian A. C., 2012, MNRAS, 427, 2998

Kakkad D. et al., 2018, A\&A, 618, A6

Kakkad D. et al., 2020, A\&A, 642, A147

Kormendy J., Ho L. C., 2013, ARA\&A, 51, 511

Kormendy J., Bender R., Cornell M. E., 2011, Nature, 469, 374

Lutz D. et al., 2020, A\&A, 633, A134

Magorrian J. et al., 1998, AJ, 115, 2285

Maiolino R., Stanga R., Salvati M., Rodriguez Espinosa J. M., 1994, A\&A, 290, 40

Martini P., Regan M. W., Mulchaey J. S., Pogge R. W., 2003a, ApJS, 146, 353

Martini P., Regan M. W., Mulchaey J. S., Pogge R. W., 2003b, ApJ, 589, 774

Mazzalay X., Rodríguez-Ardila A., Komossa S., McGregor P. J., 2013, MNRAS, 430, 2411

Miyaji T., Wilson A. S., Perez-Fournon I., 1992, ApJ, 385, 137

Mukherjee D., Bicknell G. V., Wagner A. Y., Sutherland R. S., Silk J., 2018, MNRAS, 479, 5544

Müller Sánchez F., Davies R. I., Eisenhauer F., Tacconi L. J., Genzel R., Sternberg A., 2006, A\&A, 454, 481

Müller Sánchez F., Davies R. I., Genzel R., Tacconi L. J., Eisenhauer F., Hicks E. K. S., Friedrich S., Sternberg A., 2009, ApJ, 691, 749

Mundell C. G., Holloway A. J., Pedlar A., Meaburn J., Kukula M. J., Axon D. J., 1995, MNRAS, 275, 67

Nayakshin S., Zubovas K., 2012, MNRAS, 427, 372

Nelson D. et al., 2019, MNRAS, 490, 3234

Oh K. et al., 2018, ApJS, 235, 4

Osterbrock D. E., Ferland G. J., 2006, Astrophysics of Gaseous Nebulae and Active Galactic Nuclei, 2nd edn. University Science Books, Sausalito, CA

Pillepich A. et al., 2019, MNRAS, 490, 3196

Ramos Almeida C., Piqueras López J., Villar-Martín M., Bessiere P. S., 2017, MNRAS, 470, 964

Reunanen J., Kotilainen J. K., Prieto M. A., 2002, MNRAS, 331, 154

Ricci C., Ueda Y., Koss M. J., Trakhtenbrot B., Bauer F. E., Gandhi P., 2015, ApJ, 815, L13

Riffel R. A., 2021, MNRAS, 506, 2950

Riffel R. A., Storchi-Bergmann T., 2011a, MNRAS, 411, 469

Riffel R. A., Storchi-Bergmann T., 2011b, MNRAS, 417, 2752

Riffel R., Rodríguez-Ardila A., Pastoriza M. G., 2006, A\&A, 457, 61

Riffel R. A., Storchi-Bergmann T., Winge C., McGregor P. J., Beck T., Schmitt H., 2008, MNRAS, 385, 1129

Riffel R., Pastoriza M. G., Rodríguez-Ardila A., Bonatto C., 2009, MNRAS, 400, 273

Riffel R., Rodríguez-Ardila A., Aleman I., Brotherton M. S., Pastoriza M. G., Bonatto C., Dors O. L., 2013a, MNRAS, 430, 2002

Riffel R. A., Storchi-Bergmann T., Winge C., 2013b, MNRAS, 430, 2249

Riffel R. A., Storchi-Bergmann T., Riffel R., 2014, ApJ, 780, L24

Riffel R. A., Storchi-Bergmann T., Riffel R., 2015, MNRAS, 451, 3587

Riffel R. A., Storchi-Bergmann T., Riffel R., Dahmer-Hahn L. G., Diniz M. R., Schönell A. J., Dametto N. Z., 2017, MNRAS, 470, 992

Riffel R. A. et al., 2018, MNRAS, 474, 1373

Riffel R. et al., 2019, MNRAS, 486, 3228

Riffel R. A., Storchi-Bergmann T., Zakamska N. L., Riffel R., 2020, MNRAS, 496, 4857

Riffel R. A. et al., 2021a, MNRAS, 501, L54

Riffel R. A., Bianchin M., Riffel R., Storchi-Bergmann T., Schönell A. J., Dahmer-Hahn L. G., Dametto N. Z., Diniz M. R., 2021b, MNRAS, 503, 5161

Riffel R. A. et al., 2021c, MNRAS, 504, 3265

Rodríguez-Ardila A., Riffel R., Pastoriza M. G., 2005, MNRAS, 364, 1041
Ruschel-Dutra D., de Oliveira B. D., 2020, danielrd6/ifscube v1.0. Zenodo. Available at: https://doi.org/10.5281/zenodo.3945237

Ruschel-Dutra D. et al., 2021, MNRAS, 507, 74

Schinnerer E., Eckart A., Tacconi L. J., 2000, ApJ, 533, 826

Schmitt H. R., Donley J. L., Antonucci R. R. J., Hutchings J. B., Kinney A. L., 2003, ApJS, 148, 327

Schnorr-Müller A., Storchi-Bergmann T., Nagar N. M., Robinson A., Lena D., Riffel R. A., Couto G. S., 2014, MNRAS, 437, 1708

Schnorr-Müller A., Storchi-Bergmann T., Ferrari F., Nagar N. M., 2017a, MNRAS, 466, 4370

Schnorr-Müller A., Storchi-Bergmann T., Nagar N. M., Robinson A., Lena D., 2017b, MNRAS, 471, 3888

Schönell A. J., Riffel R. A., Storchi-Bergmann T., Winge C., 2014, MNRAS, 445,414

Schönell A. J., Jr, Storchi-Bergmann T., Riffel R. A., Riffel R., 2017, MNRAS, 464, 1771

Schönell A. J., Storchi-Bergmann T., Riffel R. A., Riffel R., Bianchin M., Dahmer-Hahn L. G., Diniz M. R., Dametto N. Z., 2019, MNRAS, 485, 2054

Scoville N. Z., Hall D. N. B., Ridgway S. T., Kleinmann S. G., 1982, ApJ, 253, 136

Simões Lopes R. D., Storchi-Bergmann T., de Fátima Saraiva M., Martini P., 2007, ApJ, 655, 718

Storchi-Bergmann T., Schnorr-Müller A., 2019, Nat. Astron., 3, 48

Storchi-Bergmann T. et al., 2007, ApJ, 670, 959

Storchi-Bergmann T., McGregor P. J., Riffel R. A., Simões Lopes R., Beck T., Dopita M., 2009, MNRAS, 394, 1148

Storchi-Bergmann T., Lopes R. D. S., McGregor P. J., Riffel R. A., Beck T., Martini P., 2010, MNRAS, 402, 819

Storchi-Bergmann T. et al., 2018, ApJ, 868, 14

Tully R. B., 2015, AJ, 149, 171

van den Bosch R. C. E., 2016, ApJ, 831, 134

Veilleux S., Tully R. B., Bland-Hawthorn J., 1993, AJ, 105, 1318

Veilleux S., Maiolino R., Bolatto A. D., Aalto S., 2020, A\&AR, 28, 2

Zaritsky D., Smith R., Frenk C., White S. D. M., 1997, ApJ, 478, 39

Zubovas K., Bourne M. A., 2017, MNRAS, 468, 4956

Zubovas K., Nayakshin S., King A., Wilkinson M., 2013, MNRAS, 433, 3079

\section{SUPPORTING INFORMATION}

Supplementary data are available at MNRAS online.

Appendix A. Channel maps.

Please note: Oxford University Press is not responsible for the content or functionality of any supporting materials supplied by the authors. Any queries (other than missing material) should be directed to the corresponding author for the article.

\section{APPENDIX A: CHANNEL MAPS}

The channel maps of the [Fe II] $1.2570 \mu \mathrm{m}, \mathrm{Pa} \beta$, and $\mathrm{H}_{2} 2.1218 \mu \mathrm{m}$ for the six galaxies analysed here are presented as a supplementary file. They support the interpretation of the gas kinematics we present throughout the main body of the paper.

\section{APPENDIX B: NOTES ON INDIVIDUAL GALAXIES}

In this section, we discuss the results from Figs 1-6 in details and put our measurements in context with previous results from the literature. All the distances to the galaxies are obtained from the redshift (Riffel et al. 2018). The morphological classifications are from the Third Reference Catalogue of Bright Galaxies (RC3; de Vaucouleurs et al. 1991) and the activity types are obtained directly from the Swift-BAT 
105-month catalogue (Oh et al. 2018) unless stated otherwise. The names of the galaxies follow the same pattern of our previous works (Riffel et al. 2017, 2018, 2021b; Schönell et al. 2019), but we indicate the alternative names in each subsection.

\section{B1 NGC 788}

This is a lenticular galaxy (SA0/a?(s)), with faint spiral arms seen in the HST F606W filter image (Martini et al. 2003a), at a distance of $57 \mathrm{Mpc}$ and hosting a type 2 Seyfert (Sy2) nucleus. In Fig. 1, we present the flux, velocity, and velocity dispersion for [Fe II], $\mathrm{Pa} \beta$, and $\mathrm{H}_{2}$. The ionized gas distribution and kinematics in our maps agree with the ones shown in Schönell et al. (2019). But due to the sky line superimposed on the spectrum (see Section 3.1) we observe differences in the $\mathrm{H}_{2} 2.1218 \mu \mathrm{m}$ velocity field - ours show a larger velocity amplitude $\left(\sim 150 \mathrm{~km} \mathrm{~s}^{-1}\right)$ than theirs. The emission line flux distributions for the ionized and molecular gas are distinct. The $[\mathrm{Fe} \mathrm{II}]$ is most extended along the east-west direction with clumpy emission at the nucleus and at 0.8 arcsec east and west of it. The $\mathrm{Pa} \beta$ flux distribution is more extended along the south-west-northeast direction, which is the approximate orientation of the galaxy minor axis (Riffel et al. 2017). The $\mathrm{H}_{2}$ emission is distributed along the whole FoV with the highest fluxes observed along the northsouth direction and extending to 0.5 arcsec from each side of the nucleus, almost perpendicular to the orientation of the [Fe II] flux distribution. This feature is compatible with the dusty molecular torus detected in $\mathrm{CO}(3-2)$ line emission, perpendicular to the ionized gas winds in Seyfert galaxies as observed in the Galaxy Activity, Torus and Outflows Survey (GATOS; Garcia-Burillo et al. 2021). The velocity fields show evidence for a rotation pattern but heavily distorted. The velocity amplitude reaches $150 \mathrm{~km} \mathrm{~s}^{-1}$, which is twice the value observed for the stellar velocity field (Riffel et al. 2017). The lowest velocity dispersion values are observed for the $\mathrm{H}_{2}$, where the smaller values are in the same regions as the low $\sigma_{\star}$ patches from Riffel et al. (2017), and reaching values not higher than $80 \mathrm{~km} \mathrm{~s}^{-1}$. The highest values $\left(\approx 130 \mathrm{~km} \mathrm{~s}^{-1}\right)$ are seen for the [Fe II], while the $\mathrm{Pa} \beta$ has intermediate values of $\approx 100 \mathrm{~km} \mathrm{~s}^{-1}$. The higher velocity dispersion cospatial with the highest fluxes for the [Fe II] and distorted rotation pattern in the velocity field are indicatives of the presence of bipolar outflows. We further discuss these non-circular motions in Section 4.1.

\section{B2 NGC 3516}

NGC 3516 is a lenticular galaxy at a distance of $37 \mathrm{Mpc}$, classified as (R)SB0 $0^{0}$ ?(s), hosting a Sy1.2 nucleus, but recently the detection of an ultraviolet flare characterizes this galaxy as a changing-look AGN (Ilić et al. 2020). Fig. 2 shows its flux distributions, velocity, and velocity dispersion maps. The peak of the emission in ionized and molecular gas is located at the nucleus and the emission line flux distributions are similar to those obtained in Schönell et al. (2019). The [Fe II] emission is more extended than that of $\mathrm{Pa} \beta$ and is observed almost over the whole FoV. A $20 \mathrm{~cm}$ continuum radio emission is observed to the north of the nucleus (Miyaji, Wilson \& Perez-Fournon 1992), but unlike Barbosa et al. (2009) that observe a correlation between the [S III] $9069 \AA$ A emission and the radio, we do not see this feature in our ionized gas flux maps. The $\mathrm{H}_{2}$ emission is extended over the whole FoV and is more extended to the south-west of the nucleus that is consistent with the location of the dust lanes present there as reported by Martini et al. (2003a). The velocity fields of ionized and molecular gas show rotation patterns. The highest velocity gradients are aligned in the north-east-south-west direction that is consistent with the orientation of the stellar (Barbosa et al. 2006; Riffel et al. 2017) and [S III] (Barbosa et al. 2009) velocity fields. The direction of blueshifts and redshifts is also consistent with those observed in [O III] $\lambda 5007$ long-slit observations (Fischer et al. 2013). These similarities indicate that the gas may be located in the same plane as the stars. $\mathrm{Pa} \beta$ and $\mathrm{H}_{2}$ show the lowest velocity dispersion $\left(\sigma<150 \mathrm{~km} \mathrm{~s}^{-1}\right)$ with the smaller values observed for the former.

The $[\mathrm{Fe} I \mathrm{II}]$ presents higher velocity dispersion to the east of the nucleus consistent with the location of higher [S III] velocity dispersion (800 $\mathrm{km} \mathrm{s}^{-1}$ ) where Barbosa et al. (2009) detected an excess of blueshifts associated with an ionized gas outflow. This scenario may also apply to the [Fe II] as the velocity field deviates from the usual rotation pattern and the velocity dispersion is higher, reaching $\sim 200 \mathrm{~km} \mathrm{~s}^{-1}$. The presence of a bipolar outflow was proposed by Goad \& Gallagher (1987) and more recently by Fischer et al. (2013) who reported high [O III] velocity dispersion values $\left(600 \mathrm{~km} \mathrm{~s}^{-1}\right)$ to the north-east of the nucleus, at distances smaller than 1 arcsec. Another hypothesis to explain the complex kinematics of NGC 3516 is the presence of a twin-jet (Veilleux, Tully \& BlandHawthorn 1993) interacting with the ambient gas.

\section{B3 NGC 5506}

NGC 5506, also known as Mrk 1357, is a spiral galaxy classified as Sa pec edge-on, at a distance of $26 \mathrm{Mpc}$ and in a pair with NGC 5507 (Tully 2015). Its nucleus is classified as Sy1.9. In the top three rows of Fig. 3, we present the flux, velocity, and velocity dispersion of the narrow component of [ $\mathrm{Fe} \mathrm{II}], \mathrm{Pa} \beta$, and $\mathrm{H}_{2}$ emission lines. The narrow component for the $\mathrm{H}_{2}$ and [Fe II] emission lines shows the highest flux levels to the west and north-west of the nucleus that is consistent with the [O III] $\lambda 5007$ emission from HST long-slit observations (Fischer et al. 2013). On the other hand, the narrow component of the $\mathrm{Pa} \beta$ emission peaks $\sim 0.2$ arcsec to the east of the nucleus. The molecular gas has a similar distribution to the one presented in Schönell et al. (2019). The same does not apply to the ionized gas: their maps are more similar to our broad component maps, as a consequence of their use of Gauss-Hermite functions being more sensitive to the wings in the emission line profiles and do not disentangle the multiple kinematic components of the line profiles. The velocity fields for the narrow components show mostly a rotation pattern, with a similar orientation to that observed for the stars (Riffel et al. 2017). Distortion from rotation pattern is observed mostly in the [Fe II] velocity field (although some distortion is also seen for $\mathrm{Pa} \beta$ and $\mathrm{H}_{2}$ ) that presents an excess of blueshifts $\left(\sim-50 \mathrm{~km} \mathrm{~s}^{-1}\right)$ to the north. Enhanced $\sigma$ values $\left(>150 \mathrm{~km} \mathrm{~s}^{-1}\right)$ are seen in the same region, indicating that this structure is likely tracing the emission of outflowing gas (see Section 4.1). The outflow hypothesis is also supported by the fact that the stellar kinematics could not be mapped in the central arcsec because the $\mathrm{CO}$ absorption bands are diluted by the AGN radiation (Riffel et al. 2009, 2017) and that a biconical ionized gas outflow perpendicular to the galaxy disc is detected by Maiolino et al. (1994).

The flux, velocity, and velocity dispersion maps of the broad component of [Fe II] and $\mathrm{Pa} \beta$ are presented in the two bottom rows of Fig. 3. The fluxes for the broad components are up to one order of magnitude larger than those of the narrow components, indicating that most of the gas in NGC 5506 is disturbed - as the broader profiles are interpreted as due to such disturbances. As mentioned above, our broad component maps agree with those previously presented by Schönell et al. (2019). The velocity dispersion is larger than $300 \mathrm{~km} \mathrm{~s}^{-1}$ over the whole FoV, indicating the gas is more disturbed most probably due to the presence of gas outflows. The velocity 
maps of the broad component are also indicative of the presence of outflows. The $\operatorname{Pa} \beta$ velocity field shows redshifts over most of the FoV, except for a small blueshifted region to the north. For the [Fe II], velocities between zero and small negative values are seen at most locations, while some redshifts are observed to the south and south-east of the nucleus and some higher blueshifts are seen to the north. This pattern of velocity is consistent with the biconical outflow proposed by Fischer et al. (2013) to the north and south $\left(\mathrm{PA}=22^{\circ}\right)$ based on the [O III] emission line. These authors suggest an opening angle of $40^{\circ}$, but our velocity maps indicate this angle is larger possibly because the $[\mathrm{O} \mathrm{III}]$ emission traces the higher ionization that is more collimated.

In summary, for NGC 5506 we find that the narrow components trace a rotating disc, but excess of blueshifts to the north associated with higher velocity dispersion indicates the presence of an ionized outflow. The broad components trace only the ionized outflow.

\section{B4 NGC 3227}

NGC 3227 is a spiral galaxy (SAB(s)a) interacting with the elliptical galaxy NGC 3226 and forming the system Arp 094 (Arp 1966). Besides its companion, this galaxy resides in a group with 13 (Garcia 1993) or 14 (Crook et al. 2007) galaxies, at a distance of $16 \mathrm{Mpc}$ and hosts a Sy1.5 nucleus. The narrow component flux, velocity, and velocity dispersion maps are presented in the top three rows of Fig. 4 indicated by the green line on the right. The flux distributions of the $[\mathrm{Fe} \mathrm{II}]$ and $\mathrm{H}_{2}$ are similar: both present the emission peak at $\sim 0.3$ arcsec south-east of the nucleus and an elongated structure approximately along the direction of the line of nodes of the stellar velocity field (see Fig. 7 and Riffel et al. 2017). The morphology of the $\mathrm{H}_{2}$ emission structure within the inner $1 \operatorname{arcsec}^{2}$ is in agreement with the SINFONI observations previously presented by Davies et al. (2006) at these scales. The $\mathrm{Pa} \beta$ emission is more concentrated at the centre as already shown for the $\mathrm{Br} \gamma$ emission (Davies et al. 2006) and the total $\mathrm{Pa} \beta$ emission (Schönell et al. 2019), and shows a ring-like structure at 1 arcsec from the nucleus. This circumnuclear ring has already been detected in cold molecular gas $\mathrm{CO}(2-1)$ (Schinnerer, Eckart \& Tacconi 2000; Davies, Mark \& Sternberg 2012), with a radius of 1.5 arcsec, and in the F160W HST image after the subtraction of a model for the bulge and disc emission (Davies et al. 2006) in which the authors detect the ring at a radius of 1.7 arcsec. Higher resolution $\mathrm{CO}(2-1)$ (beam size of $0.214 \times 0.161 \operatorname{arcsec}^{2}$ ) ALMA observations unravelled the structure of the ring: several star-forming clumps associated with star formation events (AlonsoHerrero et al. 2019). The presence of a ring of star-forming regions is also supported by the low $\mathrm{H}_{2} / \mathrm{Br} \gamma$ ratios (Schönell et al. 2019; Riffel et al. 2021c), higher $\mathrm{Br} \gamma$ equivalent width (Riffel et al. 2021c), low gas ( $\sim 50 \mathrm{~km} \mathrm{~s}^{-1}-$ Fig. 4), and stellar velocity dispersion (Barbosa et al. 2006; Riffel et al. 2017) values.

The ionized and molecular gas velocity fields show amplitudes of $150 \mathrm{~km} \mathrm{~s}^{-1}$ and signatures of a rotation pattern that, as for the other galaxies, is distorted indicating the presence of non-circular motions. The velocity gradient is oriented along the stellar line of the nodes (see Fig. 7). Our $\mathrm{H}_{2}$ velocity field agrees with that of the higher resolution $(0.085 \mathrm{arcsec})$ and smaller FoV $\left(0.8 \times 0.8 \operatorname{arcsec}^{2}\right) K$ band SINFONI data obtained by Davies et al. (2006), who proposed that velocity asymmetries along the galaxy minor axis can be caused by gas outflows. Recently, Alonso-Herrero et al. (2019), analysing the $\mathrm{CO}(2-1)$ kinematics, showed that streaming motions due to inflows associated with the large-scale bar and circumnuclear starforming ring are the cause of the distortion in the molecular gas velocity field. These authors also interpret the $\mathrm{CO}(2-1)$ kinematics as being consistent with the presence of outflows at scales of $\sim 70$ pc along the direction of the minor axis of the nuclear disc $\left(\mathrm{PA}=50^{\circ}\right)$. The $\mathrm{Pa} \beta$ and the $[\mathrm{Fe} \mathrm{II}]$ profiles have higher velocity dispersion values $\left(>100 \mathrm{~km} \mathrm{~s}^{-1}\right)$ distributed in a 0.5 arcsec wide arcshaped structure inside the star-forming ring. This enhanced velocity dispersion structure suggests that a spherical-shaped gas outflow might be present in NGC 3227. The $\mathrm{H}_{2}$ velocity dispersion is higher $\left(\sim 120 \mathrm{~km} \mathrm{~s}^{-1}\right)$ in a region perpendicular to the highest flux values and cospatial with the distortion observed in the velocity field. Davies et al. (2006) interpret this feature as due to the fact that the $\mathrm{H}_{2}$ is located in a thick disc instead of in a thin stellar disc. This scenario is also supported by the ALMA data from Alonso-Herrero et al. (2019) that interpret the nuclear emission of NGC 3227 as due to molecular gas in a disc that is extended along tens of parsecs instead of in a compact torus.

On the two bottom rows of Fig. 4, we present the flux distribution, velocity, and velocity dispersion for the $[\mathrm{Fe} I I]$ and $\mathrm{Pa} \beta$ broad components. The highest fluxes are somewhat extended to the northnorth-east. Also, as the broad component is overall brighter than the narrow component, the maps based on the fitting of the line profiles by a single Gauss-Hermite series shown in Schönell et al. (2019) are very similar to ours. One of the peaks of the radio emission in $18 \mathrm{~cm}$ detected by Mundell et al. (1995) is offset 0.4 arcsec to the north of the nucleus, which is cospatial with the elongated structure in the [Fe II] flux distribution. Also, the highest [Fe II] velocity dispersion values $\left(250 \mathrm{~km} \mathrm{~s}^{-1}\right)$ are seen at this same location indicating that the gas is possibly being disturbed by the radio source. The velocity fields show only blueshifts, although the amplitudes are different: $150 \mathrm{~km} \mathrm{~s}^{-1}$ for $\mathrm{Pa} \beta$ and $250 \mathrm{~km} \mathrm{~s}^{-1}$ for [Fe II]. The velocity dispersion of both emission lines is higher than $200 \mathrm{~km} \mathrm{~s}^{-1}$ with the highest values observed to the north of the nucleus for [Fe II]. Barbosa et al. (2009) associate a compact radio emission at $3.6 \mathrm{~cm}$, also extended to the north, to the [S III] $9069 \AA$ A outflows. The [S III] outflows are observed as blueshifts at $\mathrm{PA}=-10^{\circ}$, which is similar to the orientation of our velocity fields, especially that for [Fe II].

In summary, the narrow component in NGC 3327 is mostly dominated by rotation, but signatures of non-circular motions are also present. The circumnuclear ring seen in the $\mathrm{Pa} \beta$ emission is cospatial with previous ${ }^{12} \mathrm{CO}$ observations and seems to surround a spherical-bubble outflow. The broad component is tracing solely the ionized gas outflow.

\section{B5 NGC 5899}

NGC 5899 at a distance of 36 Mpc makes a pair with NGC 5900 (Zaritsky et al. 1997). It is a spiral galaxy (SAB(rs)c) that hosts a Sy2 nucleus. In the top three rows of Fig. 5, we present the maps for the narrow component, as indicated by the green line on the right. The $\mathrm{H}_{2}$ flux distribution is elongated in the direction of the galaxy major axis $\left(\mathrm{PA}=25^{\circ}\right)$ and consistent with the flux map presented in Schönell et al. (2019). The flux distribution of the $\mathrm{Pa} \beta$ and [Fe II] is elongated along the north-south direction with two emission knots observed at 0.3 arcsec north and south of the nucleus. This orientation is consistent with the photometric major axis (Ruschel-Dutra et al. 2021). The $\mathrm{H}_{2}$ velocity field shows a rotation pattern but its gradient seems to be slightly displaced from the orientation of the stellar line of the nodes (see Fig. 7). The $\mathrm{H}_{2}$ velocity dispersion shows overall low values $\left(<100 \mathrm{~km} \mathrm{~s}^{-1}\right)$ with the lowest values are seen to the north and the south of the nucleus and the highest in the perpendicular direction. Excess blueshifts, observed to the northwest of the nucleus, combined with the low velocity dispersion $\left(\sim 60 \mathrm{~km} \mathrm{~s}^{-1}\right)$ of the region, may indicate the presence of streaming 
motions towards the centre of the galaxy (see Section 4.1). Besides the distinct flux distributions, the velocity fields of $\mathrm{Pa} \beta$ and [Fe II] also differ from that of $\mathrm{H}_{2}$ : blueshifts are observed mostly to the south and redshifts to the north, being opposite to the orientation of the molecular gas velocity field. In addition, the velocity dispersion at the locations of the highest flux structures is larger than that of $\mathrm{H}_{2}$. These differences indicate that the narrow components for the ionized gas are not originated from the gas in the disc, what seems to be the case for the molecular gas. Some emission of gas located in the galactic plane is seen to the north-east, as indicated by the lower velocity dispersion values and similar velocity amplitudes for the ionized and molecular gas.

The flux distributions, velocity, and velocity dispersion for the broad component are presented in the two bottom rows of Fig. 5. The flux maps of the $\mathrm{H}_{2}, \mathrm{~Pa} \beta$, and [Fe II] are similar: distributed along the north-south direction with the peak of the emission at $\approx 0.3 \operatorname{arcsec}$ south of the nucleus. The emission is also more compact than for the narrow component. The $\mathrm{H}_{2}$ velocity field shows only blueshifts at $\sim-50 \mathrm{~km} \mathrm{~s}^{-1}$ and velocity dispersion of $\sim 250 \mathrm{~km} \mathrm{~s}^{-1}$, which is lower than that of the broad components of the ionized gas emission lines. The $\mathrm{Pa} \beta$ and [Fe II] velocity fields for the broad component show mostly blueshifts, but some very low velocity redshifts $\left(\sim 30 \mathrm{~km} \mathrm{~s}^{-1}\right)$ are seen to the north of the nucleus in the [Fe II] velocity field. Their velocity dispersion maps present overall high values $\left(>250 \mathrm{~km} \mathrm{~s}^{-1}\right)$ with the highest ones observed to the north of the nucleus. This suggests that, as for the narrow component, the broad component in the ionized gas emission lines is not produced by gas in the galaxy disc, and both narrow and broad components trace an outflow that seems to have a bipolar geometry with its axis oriented along the north-south direction. The blueshifts probably trace the front wall of a cone-like outflow, the zero velocity spaxels the part that is aligned with the plane of the sky, and redshifts the back of the cone. These features lead us to suggest that the $\mathrm{H}_{2}$ broad emission is produced by the interaction between the ionized outflow and the molecular gas in the disc.

In summary, for NGC 5899 the ionized gas kinematics seems to be dominated by a bipolar outflow, oriented approximately along the north-south direction. This outflow interacts with gas in the disc, pushing away molecular gas, which produces the broad $\mathrm{H}_{2}$ component. Most of the molecular gas seems to be located in the disc of the galaxy.

\section{B6 Mrk 607}

Mrk 607, also known as NGC 1320 and MCG-01-09-036, is an edge-on spiral galaxy with inclination $i=70^{\circ}$ (Riffel et al. 2017), classified as Sa?, hosts a Sy2 nucleus, and at a distance of $\approx 37 \mathrm{Mpc}$. It is a member of a small group of galaxies, together with NGC 1321 and NGC 1289 (Crook et al. 2007). In the top subfigure of Fig. 6, we present the flux distribution, velocity, and velocity dispersion for what we call the disc component The flux distributions for the molecular and ionized gas are elongated along the north-west-southeast direction, approximately the direction of the galaxy major axis, $\mathrm{PA}=138^{\circ}$ (Riffel et al. 2017). The highest flux values are observed at and near the nucleus for the ionized gas, but for the $\mathrm{H}_{2}$ the flux distribution is more homogeneous over the whole FoV. Similar extensions and orientations are observed in the flux distributions of optical emission lines, for instance [O III] $\lambda 5007, \mathrm{H} \alpha$, [N II] $\lambda 6583$, and [S II] $\lambda 6731$ (Freitas et al. 2018). Our flux distributions are also similar to the HST [O III] flux distribution (Schmitt et al. 2003) where the strongest emission is observed at the nucleus and extended to up to 3.75 arcsec to the north-west, in the direction of the galaxy major axis, which is consistent with the $\mathrm{Pa} \beta$ flux distribution. The velocity fields present a well-defined rotation pattern, with a velocity amplitude of $\sim 200 \mathrm{~km} \mathrm{~s}^{-1}$. Although the gradient of the stellar velocity field (Riffel et al. 2017) is oriented in the same direction, the gas is rotating in the opposite direction. This result was also previously reported by Freitas et al. (2018). The counter-rotation between gas and stars suggests that these components have different origins. Since the stellar disc is more stable than the gas, and thus the orbits of stars are harder to change, it is likely that the gas kinematics has been affected by the interaction between Mrk 607 and its closest companion, NGC 1321.

In the two sets of panels in the bottom of Fig. 6, we present the maps for the blueshift component, to the left, and for the redshift component, to the right. As the region fitted with three Gaussian functions corresponds only to the central arcsecond or so we zoomed in the central $1 \times 1 \operatorname{arcsec}^{2}$ region of the FoV shown as the green square in the $\mathrm{H}_{2}$ flux map of the disc component. A similar kinematic pattern (with one blueshifted and one redshifted component) around the nucleus has been observed in other Seyfert galaxies, e.g. NGC 5929 (Riffel et al. 2015), interpreted as being produced by an equatorial outflow, i.e. the torus itself expanding outwards. This interpretation is proposed because the highest velocity dispersion values - or the two components here - are observed perpendicular to the observed radio emission axis. Previous optical IFS of Mrk 607 also shows an enhancement in the gas velocity dispersion perpendicularly to the galaxy disc (Freitas et al. 2018), while the radio continuum emission is extended along the northsouth direction, with some faint emission also to the north-west along the major axis (Colbert et al. 1996). The beam size of this observation is given by a Gaussian with FWHM of 3 arcsec, the size of our FoV, thus a clear association between the jet orientation and the outflow cannot be made. The peak of the flux distributions of the blueshifted and redshifted components is slightly displaced from the nucleus: the blueshifted component emission peaks north-west of the nucleus, while the redshifted emission peaks south-east of the nucleus.

In summary, for Mrk 607, both the ionized and molecular gas present two kinematic components: (i) one due to emission of gas rotating in the galaxy disc, in the opposite direction of the stellar motions; and (ii) an equatorial outflow or compressed gas by wide-opening angle outflows in colder gas phases, identified by the blueshifted and redshifted emission line components.

This paper has been typeset from a $\mathrm{T}_{\mathrm{E}} \mathrm{X} / \mathrm{LAT}_{\mathrm{E}} \mathrm{X}$ file prepared by the author. 